\title{
Lethe: A Tunable Delete-Aware LSM Engine
}

\author{
Subhadeep Sarkar, Tarikul Islam Papon, Dimitris Staratzis, Manos Athanassoulis \\ Boston University \\ \{ssarkar1, papon, dstara, mathan\}@bu.edu
}

\begin{abstract}
Data-intensive applications fueled the evolution of log structured merge (LSM) based key-value engines that employ the out-of-place paradigm to support high ingestion rates with low read/write interference. These benefits, however, come at the cost of treating deletes as a second-class citizen. A delete inserts a tombstone that invalidates older instances of the deleted key. State-of-the-art LSM engines do not provide guarantees as to how fast a tombstone will propagate to persist the deletion. Further, LSM engines only support deletion on the sort key. To delete on another attribute (e.g., timestamp), the entire tree is read and re-written. We highlight that fast persistent deletion without affecting read performance is key to support: (i) streaming systems operating on a window of data, (ii) privacy with latency guarantees on the right-to-be-forgotten, and (iii) en masse cloud deployment of data systems that makes storage a precious resource.

To address these challenges, in this paper, we build a new key-value storage engine, Lethe, that uses a very small amount of additional metadata, a set of new delete-aware compaction policies, and a new physical data layout that weaves the sort and the delete key order. We show that Lethe supports any user-defined threshold for the delete persistence latency offering higher read throughput $(1.17-1.4 \times)$ and lower space amplification $(2.1-9.8 \times)$, with a modest increase in write amplification (between $4 \%$ and $25 \%$ ). In addition, Lethe supports efficient range deletes on a secondary delete key by dropping entire data pages without sacrificing read performance nor employing a costly full tree merge.
\end{abstract}

\section{INTRODUCTION}

Systems are Optimized for Fast Data Ingestion. Modern data systems process an unprecedented amount of data generated by a variety of applications that include data analytics,

Permission to make digital or hard copies of all or part of this work for personal or classroom use is granted without fee provided that copies are not made or distributed for profit or commercial advantage and that copies bear this notice and the full citation on the first page. Copyrights for components of this work owned by others than ACM must be honored. Abstracting with credit is permitted. To copy otherwise, or republish, to post on servers or to redistribute to lists, requires prior specific permission and/or a fee. Request permissions from permissions@acm.org.

SIGMOD'20, fune 14-19, 2020, Portland, OR, USA

(c) 2020 Association for Computing Machinery.

ACM ISBN 978-1-4503-6735-6/20/06 ..\$15.00

https://doi.org/10.1145/3318464.3389757
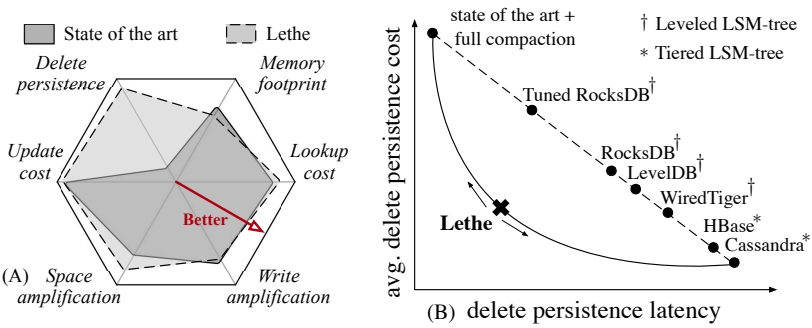

Figure 1: (A) Lethe strikes an optimal balance between the latency/performance for timely delete persistence in LSM-trees, and (B) supports timely delete persistence by navigating the latency/cost trade-off.

stream processing, Internet of Things and 5G [18, 30]. Cloudbased latency-sensitive applications like live video streaming [40], real-time health monitoring [55], e-commerce transactions [39], social network analysis [59], and online gaming [49], generate large volumes of data at a high velocity that requires hybrid transactional/analytical processing (HTAP) $[7,51,54]$. Thus, for the past decade, one of the main data management research challenges has been to design data systems that can sustain fast data ingestion rate and process queries at low latency $[3,9,54]$. To achieve this, modern data stores reduce read/write interference by employing out-of-place ingestion [11, 12, 21, 38, 44, 46, 56, 63].

LSM-based Key-Value Stores. The classical out-of-place design is the log-structured merge (LSM) tree. LSM-trees buffer incoming data entries in main memory, and periodically flush this buffer as an immutable sorted run on durable storage [21, 50, 53, 57, 64]. In turn, as more sorted runs accumulate, they are iteratively sort-merged to form fewer yet larger sorted runs. This process, termed compaction, reduces the number of sorted runs accessed during a read query with amortized merging cost. Every compaction sort-merges existing sorted runs from consecutive levels and discards any invalid entries. LSM-trees are adopted by several modern systems including LevelDB [36] and BigTable [17] at Google, RocksDB [29] at Facebook, X-Engine [39] at Alibaba, Voldemort [48] at LinkedIn, Dynamo [25] at Amazon, Cassandra [5], HBase [6], and Accumulo [4] at Apache, and bLSM [61] and cLSM [35] at Yahoo. Relational data systems have been increasingly adopting LSM-style of updates. MyRocks [28] uses RocksDB as storage engine and SQLite4 [62] has experimented with LSM-trees in its storage layer, while columnar systems use LSM-style updates $[20,44,63,70]$. 
The Challenge: Out-of-place Deletes. LSM engines use the out-of-place paradigm for any write operation, including ingestion (inserts), modification (updates), and deletion (deletes). As a result, a delete (update) is implemented by inserting additional meta-data that logically invalidates the older target entries [16]. We refer to this process as logical deletes (updates). Both logical deletes and updates show a complex three-way trade-off [13], however, logical deletes have wider implications in terms of (i) space amplification, (ii) read cost, (iii) write amplification, and (iv) privacy considerations, and hence, is the primary focus of this work.

In particular, a logical delete, inserts a tombstone that invalidates all the older entries for a given key, with the expectation that, eventually, they will be persistently deleted. In practice, the delete persistence latency is driven by (a) the system design choices and (b) workload characteristics. Neither can be fully controlled during execution, therefore, providing latency guarantees for persistent deletion in state-of-the-art LSM engines is nearly impossible. In fact, LSM-trees have a potentially unbounded delete persistence latency. In order to limit it, current designs employ a costly full-tree compaction that interferes with read performance and write amplification, and results in performance unpredictability [39].

Deletes in LSM-trees. LSM-trees are employed as the storage layer for relational systems [28], streaming systems [2, $41,65]$, and pure key-value storage [52, 68]. As a result, an LSM delete operation may be triggered by various logical operations, not limited to user-driven deletes. For example, deletes are triggered by workloads that involve periodic data migration [58], streaming operations on a running window [39, 43], or entail cleanup during data migration [58]. In particular, dropping tables in an LSM-tree with multiple column families is realized through a range delete operation [58]. Another frequent example is data collections sorted on creation timestamp. In that case, a classical out-of-place update is not enough since the key (timestamp) will also change. Hence, every update translates to a delete followed by the insertion of the new version [15]. Below, we distill the common concepts of two frequent delete use-cases.

Scenario 1: An e-commerce company EComp stores its order details sorted by order_id in an LSM-tree, and needs to delete the order history for a particular user. Within the system, this delete request is translated to a set of point and range deletes on the sort key, i.e., order_id.

Scenario 2: A data company DComp stores its operational data in an LSM-tree with document_id as the sort key. As most of the data are relevant only for $D$ days, DComp wants to delete all data with a timestamp that is older than $D$ days (and archive them). At the same time, DComp frequently accesses the documents using document_id, hence, the sort key (document_id) is different from the delete key (timestamp).
Why State of the Art is not Enough? LSM engines cannot efficiently support EComp from the first scenario for two reasons. First, as deletes insert tombstones (retaining the physical entries), they increase space amplification. Second, retaining superfluous entries in the tree adversely affects read performance because read queries have to discard potentially large collections of invalid entries, which further "pollute" the filter metadata [39], and increase write amplification because invalid entries are repeatedly compacted. Further, LSM engines are ill-suited for DComp from the second scenario because they cannot efficiently support a range deletion in a delete key other than the sort key (termed secondary range deletes). Instead, they employ a full tree compaction, which causes an excessive number of wasteful I/Os while reading, merging, and re-writing the sorted files of the entire tree [39].

Delete persistence latency. In order to be able to report that a delete persisted, the corresponding tombstone has to reach the last level of the tree through iterative compactions to discard all invalidated entries. The time elapsed between the insertion of the tombstone in the tree and the completion of the last-level compaction is termed delete persistence latency. LSM logical deletes do not provide delete persistence latency guarantees, hence EComp cannot offer such guarantees to its users. In order to add a hard limit on delete persistence latency, current designs employ a costly full tree compaction as well.

Deletion as privacy. Having unbounded delete persistence latency may lead to a breach of privacy. For example, it was recently reported that Twitter retains user messages years after they have been deleted, even after user accounts have been deactivated [67]. With the new data privacy protection acts like GDPR [34] and CCPA [1], the end-to-end data lifecycle has new privacy challenges to address $[26,60]$. With user-rights, such as the right-to-be-forgotten coming into play, persistent deletion within a fixed threshold is critical. "Full tree compactions should be avoided."

In our interactions with engineers working on LSM-based production systems, we learned that periodic deletes of a large fraction of data based on timestamp are very frequent. To quote an engineer working on XEngine [39], "Applications may keep data for different durations (e.g., 7 or 30 days) for their own purposes. But they all have this requirement for deletes every day. For example, they may keep data for 30 days, and daily delete data that turned 31-days old, effectively purging 1/30 of the database every day." This deletion is performed with a full tree compaction. To further quote the same team, "Forcing compactions to set a delete latency threshold, leads to significant increase in compaction frequency, and the observed I/O utilization often peaks. This quickly introduces performance pains." For large data companies, deleting $1 / 7$ or $1 / 30$ of their database, accounts for several GBs or 
TBs that is required to be persistently removed daily. The current approach of employing full-tree compactions is suboptimal as it (1) causes high latency spikes, and (2) increases write amplification. The goal of this work is to address these challenges while retaining the benefits of LSM design.

The Solution: Lethe. We propose Lethe ${ }^{1}$, a new LSM-based key-value store that offers efficient deletes without compromising the benefits of LSM-trees. Lethe pushes the boundary of the traditional LSM design space by adding delete persistence as a new design goal, and is able to meet user requirements for delete persistence latency. Figures 1 (A) and (B) show a qualitative comparison between state-of-the-art LSM engines [5, 6, 29, 36, 68] and Lethe with respect to the efficiency and cost of timely persistent deletes. Lethe introduces two new LSM design components: FADE and KiWi.

$F A D E$ (Fast Deletion) is a new family of compaction strategies that prioritize files for compaction based on (a) the number of invalidated entries contained, (b) the age of the oldest tombstone, and (c) the range overlap with other files. FADE uses this information to decide when to trigger a compaction on which files, to purge invalid entries within a threshold.

$\mathrm{KiWi}$ (Key Weaving Storage Layout) is a new continuum of physical layouts that allows for tunable secondary range deletes without causing latency spikes, by introducing the notion of delete tiles. An LSM-tree level consists of several sorted files that logically form a sorted run. KiWi augments the design of each file with several delete tiles, each containing several data pages. A delete tile is sorted on the secondary (delete) key, while each data page remains internally sorted on the sort key. Having Bloom filters at the page level, and fence pointers for both the sort key and the secondary delete key, KiWi facilitates secondary range deletes by dropping entire pages from the delete tiles, with a constant factor increase in false positives. Maintaining the pages sorted on the sort key also means that once a page is in memory, read queries maintain the same efficiency as the state of the art.

Putting everything together, Lethe is the first LSM engine to our knowledge that offers efficient deletes while improving read performance, supports user-defined delete latency thresholds, and enables practical secondary range deletes.

Contributions. Our contributions are as follows:

- We analyze out-of-place deletes w.r.t. read performance, space and write amplification, and user privacy.

- We introduce FADE that bounds delete persistence latency without hurting performance and resource consumption.

- We introduce Key Weaving Storage Layout, the first layout that supports efficient secondary range deletes.

- We present the design of Lethe that integrates FADE and $\mathrm{KiWi}$ in a state-of-the-art LSM engine and enables fast

\footnotetext{
${ }^{1}$ Lethe, the Greek mythological river of oblivion, signifies efficient deletion.
}

deletes with a tunable balance between delete persistence latency and the overall performance of the system.

- We demonstrate that Lethe offers delete latency guarantees, having up to $1.4 \times$ higher read throughput. The higher read throughput is attributed to the significantly lower space amplification (up to $9.8 \times$ for only $10 \%$ deletes) because it purges invalid entries faster. These benefits come at the cost of 4\%-25\% higher write amplification.

- Finally, we demonstrate that Lethe is the first LSM engine to support efficient secondary range deletes at the expense of increased read cost, and we show how to tune Lethe to amortize this cost based on the workload.

\section{LSM BACKGROUND}

Basics. LSM-trees store key-value pairs, where a key refers to a unique object identifier, and the data associated with it, is referred to as value. For relational data, the primary key acts as the key, and the remaining attributes in a tuple constitute the value. As entries are sorted and accessed by the key, we refer to it as the sort key. For an LSM-tree with $L$ levels, we assume that its first level (Level 0 ) is an in-memory buffer and the remaining levels (Level 1 to $L-1$ ) are disk-resident. We adopt notation from the literature $[21,50]$.

Buffering Inserts and Updates. Inserts, updates, or deletes are buffered in memory. A delete (update) to a key that exists in the buffer, deletes (replaces) the older key in-place, otherwise the delete (update) remains in memory to invalidate any existing instances of the key on the disk-resident part of the tree. Once the buffer reaches its capacity, the entries are sorted by key to form an immutable sorted run and are flushed to the first disk-level (Level 1). When a disk-level reaches its capacity, all runs within that level are sort-merged and pushed to the next level. To bound the number of levels in a tree, runs are arranged in exponentially growing levels on disk. The capacity of Level $i(i \geq 1)$ is greater than that of Level $i-1$ by a factor of $T$, termed the size ratio of the tree. Compaction Policies: Leveling and Tiering. Classically, LSM-trees support two merging policies: leveling and tiering. In leveling, each level may have at most one run, and every time a run in Level $i-1(i \geq 1)$ is moved to Level $i$, it is greedily sort-merged with the run from Level $i$, if it exists. With tiering, every level must accumulate $T$ runs before they are sort-merged. During a sort-merge (compaction), entries with a matching key are consolidated and only the most recent valid entry is retained [27, 53]. Recently hybrid compaction policies fuse leveling and tiering in a single tree to strike a balance between the read and write throughput based on workload specifications [23, 24].

Partial Compaction. To amortize latency spikes from compactions in larger levels, state-of-the-art LSM engines organize runs into smaller files, and perform compactions at the 
granularity of files instead of levels [27]. If Level $i$ grows beyond a threshold, a compaction is triggered and one file from Level $i$ is chosen to be partially compacted with files from Level $i+1$ that have an overlapping key-range. Deciding which file to compact depends on the storage engine design. For instance, to optimize write throughput, we select files from Level $i$ with minimal overlap with files in Level $i+1$, to minimize both write amplification and compaction time.

Querying LSM-Trees. A point lookup begins at the memory buffer and traverses the tree from the smallest disk-level to the largest one. For tiering, within a level, a lookup moves from the most to the least recent tier. The lookup terminates when it finds the first matching entry. A range lookup returns the most recent versions of the target keys by sort-merging the qualifying key ranges across all runs in the tree.

Optimizing Lookups. Read performance is optimized using Bloom filters (BFs) and fence pointers. In the worst case, a lookup needs to probe every run. To reduce this cost, LSM engines use one BF per run in main memory [21, 29]. Bloom filters allow a lookup to skip probing a run altogether if the filter-lookup returns negative. In practice, for efficient storage, BFs are maintained at the granularity of files [27]. Fence pointers store the smallest key per disk page in memory [21], to quickly identify which page(s) to read for a lookup, and perform up to one $\mathrm{I} / \mathrm{O}$ per run for point lookups.

\section{THE IMPACT OF DELETES}

We now describe the design space of deletes in LSM-trees.

\subsection{Delete Design Space}

In LSM-trees an entry at Level $i$ is always more recent than an entry with the same key at Level $j$, if $j>i$. LSM-trees exploit this to logically delete using tombstones that supersede older entries with a matching key. The left part of Figure 3 shows a leveled LSM-tree, the structure of a key-value pair, and a tombstone. A key-value contains typically many attributes as part of the value, and a tombstone consists of the (deleted) key and the tombstone flag.

3.1.1 Primary Deletes. We discuss deletes on the sort key. Point Deletes insert a tombstone against the key to be deleted (Figure 2). Within memory buffer, the tombstone replaces in-place any older entry with a matching key. On disk, the tombstones are stored within a run in sorted order along with other key-value pairs. During compaction, a tombstone deletes older entries with the same key and is retained as there might be more (older) entries with the same delete key in subsequent compactions (Fig. 2). A tombstone is discarded during its compaction with the last level of the tree, making the logical delete persistent.

Range Deletes in LSM-trees are common, however, they cause performance problems. Range deletes generate special

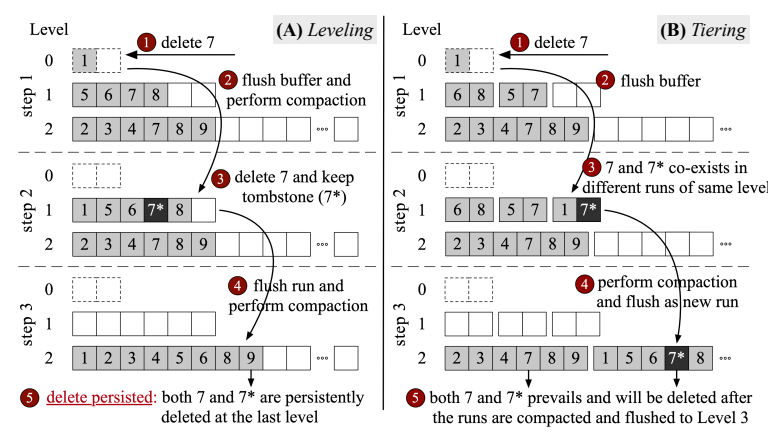

Figure 2: In an LSM-tree, for every tombstone, there can be (A) one matching entry per level for leveling or (B) one matching entry per tier per level ( $T$ per level) for tiering, where $T=3$ in this example.

range tombstones that are stored in a separate range tombstone block within files [29]. During data access, a histogram storing deleted ranges is maintained in memory which has to be accessed by every point query slowing down read accesses $[15,58]$. Similar to point deletes, range deletes are persisted when the files that contain them are compacted with the last level, leaving potentially unbounded persistence latency. Thus, in practice, a complete full tree compaction is periodically employed to ensure delete persistence [39]. During such compactions, all reads and writes to the data store are stalled, which results in latency spikes.

Persistence Latency. The latency for persisting a logical delete depends on the workload and the data size. The middle part of Figure 3 illustrates the operation "delete all entries with $\mathrm{ID}=\mathrm{k}$ ". Within the system, the operation inserts a tombstone, $\mathrm{k}^{*}$, that logically invalidates $\mathrm{k}$. On disk, entries with key $\mathrm{k}$ may be located at any level between 1 and $L$. Thus, to ensure delete persistence, $\mathrm{k}^{*}$ must participate in $L$ compactions, one at each level of the tree. Since compactions are triggered when a level reaches a nominal capacity, the rate of unique insertions is effectively driving the compactions. The size of a level grows exponentially with $T$, therefore, a taller tree requires exponentially more unique insertions to propagate a tombstone to the last level. Hence, the delete persistence latency depends on (i) the rate of unique insertions and (ii) the current height of a tree.

Adversarial Workloads. Tombstones may be recycled in intermediate levels of the tree leading to unbounded delete persistence latency and perpetual retention of invalid entries [15]. For example, a workload that mostly modifies hot data (in the first few levels) will grow the tree very slowly, keeping its structure mostly static. Another example is a workload with interleaved inserts and deletes, with the deletes issued on a few recently inserted entries that are at the smaller levels. In both cases, a newly inserted tombstone may be recycled in compactions high up the tree that consolidate entries rather than propagate towards the last level. 

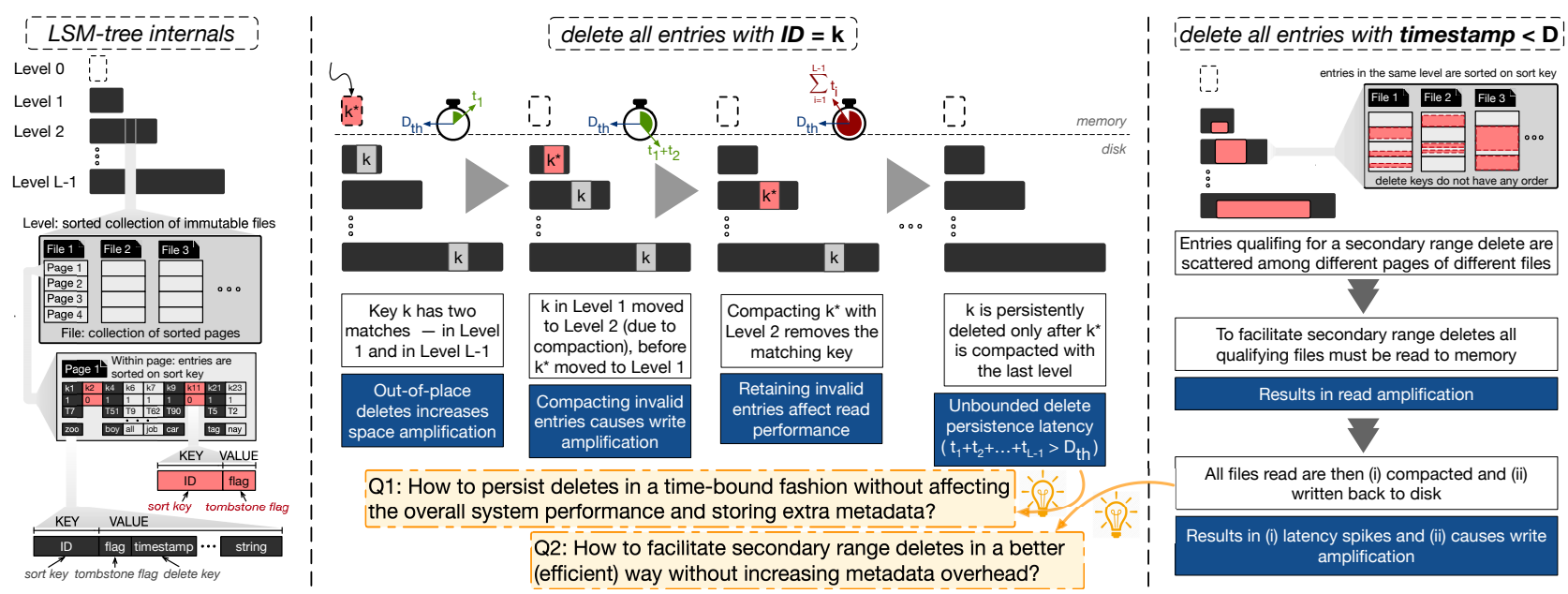

Figure 3: The design space of deletes in LSM-trees.

3.1.2 Secondary Deletes. We refer to deletes based on an attribute other than the sort key as secondary deletes. The most common type of secondary deletes is a secondary range delete. Consider the operation "delete all entries that are older than $D$ days", similar to the second scenario from the introduction. In the left part of Figure 3, we highlight the sort key (ID) and the delete key (timestamp) of a key-value pair. As the entries in a tree are sorted on the sort key, an entry with a qualifying delete key may be anywhere in the tree, and this delete pattern is not efficiently supported.

3.1.3 Limitations of the State of the Art. In state-of-theart LSM engines, deletes are considered as "second-class citizens". In practice, to ensure time-bounded persistence of logical deletes and to facilitate secondary range deletes, data stores resort to periodic full-tree compaction [19, 39]. However, this is an extremely expensive solution as it involves superfluous disk I/Os, increases write amplification and results in latency spikes. To reduce excessive I/Os, RocksDB implements a file selection policy based on the number of tombstones [29]. This reduces the amount of invalid entries, but it does not offer persistent delete latency guarantees.

\subsection{Implications of Out-of-place Deletes}

Next, we quantify the implications of out-of-place deletes on read performance, and space and write amplification.

Model Details. We assume an LSM-tree with size ratio $T$, that stores $N$ entries across $L+1$ levels. The size of the memory buffer is $M=P \cdot B \cdot E$, where $P$ is the number of disk pages in the buffer, $B$ is the number of entries per page, and $E$ is the average size of an entry. The capacity of this tree is $\sum_{i=0}^{L} M \cdot T^{i}$, where $M \cdot T^{i}$ is the capacity of Level $i$. The $N$ entries inserted in the tree includes $\delta_{p}$ point tombstones and $\delta_{r}$ range tombstones that have an average selective of $\sigma$. Table 1 shows all the parameters used in our modeling.
3.2.1 Space Amplification. Deletes increase space amplification by (i) the tombstones and (ii) the invalidated entries (for every key, there might be several invalid versions). Space amplification increases storage cost and the overhead for data organization (sorting) and processing (read I/Os during compaction). Commercial databases often report space amplification of about $11 \%$ [58], however, this corresponds to $T=10$, a single point in the vast design continuum.

Analysis. Following prior work [23], we define space amplification as the ratio between the size of superfluous entries and the size of the unique entries in the tree, $s_{a m p}=$ $\frac{\operatorname{csize}(N)-\operatorname{csize}(U)}{\operatorname{csize}(U)}$, where $\operatorname{csize}(N)$ is the cumulative size of all entries and $\operatorname{csize}(U)$ is the cumulative size of all unique entries. Note that $s_{a m p} \in[0, \infty)$, and that if all inserted keys are unique there is no space amplification.

Without Deletes. Assume a workload with inserts and updates (but no deletes) for a leveled LSM-tree. In the worst case, all entries in levels up to $L-1$ can be updates for the entries in Level $L$, leading to space amplification $O(1 / T)$. For a tiered LSM-tree, the worst case is when the tiers of a level overlap, and the first $L-1$ levels contain updates for Level $L$. This leads to space amplification $O(T)$.

With Deletes. If the size of a tombstone is the same as the size of a key-value entry, the asymptotic worst-case space amplification remains the same as that with updates for leveling. However, in practice, a tombstone is orders of magnitude smaller than a key-value entry. We introduce the tombstone size ratio $\lambda=\frac{\text { size (tombstone })}{\text { size (key-value })} \approx \frac{\text { size }(k e y)}{\operatorname{size}(\text { key })+\operatorname{size}(v a l u e)}$, where size(key) and size(value) is the average size of a key and an entry, respectively. $\lambda$ is bounded by $(0,1]$, and a smaller $\lambda$ implies that a few bytes (for tombstones) can invalidate more bytes (for key-values) and lead to larger space amplification given by $O\left(\frac{(1-\lambda) \cdot N+1}{\lambda \cdot T}\right)$. For tiering, in the worst case, 
tombstones in the recent-most tier can invalidate all entries in that level, resulting in space amplification $O\left(\frac{N}{1-\lambda}\right)$.

3.2.2 Read Performance. Point tombstones are hashed to the BFs the same way as valid keys, and thus, increase the false positive rate (FPR) for the filters as well as the I/O cost for point lookups. Also, deleted entries cause range queries to scan invalid data before finding qualifying keys. Consider that a range delete with $0.5 \%$ selectivity over a 100GB database invalidates $500 \mathrm{MB}$, which might have to be scanned (and discarded) during query execution.

Analysis: Point Lookups. A point lookup probes one (or more) BF before performing any disk I/O. The FPR of a BF depends on the number of bits allocated to the filter in the memory $(m)$ and the number of entries $(N)$ hashed into the filter, and is given by $e^{-m / N \cdot(\ln (2))^{2}}$. For leveling, the average worst-case point lookup cost on non-existing entries is $O\left(e^{-m / N}\right)$, and for tiering, the cost becomes $O\left(T \cdot e^{-m / N}\right)[21]$ For lookups on existing entries, this cost increases by 1 as the lookup has to probe at least one page. Since tombstones are hashed into the BFs, retaining tombstones and invalid entries increases their FPR, thus hurting point lookups.

Analysis: Range Lookups. A range query on the sort key reads and merges all qualifying disk pages. The I/O cost of a short range query accessing one page per level is $O(L)$ for leveling and $O(L \cdot T)$ for tiering. The $\mathrm{I} / \mathrm{O}$ cost for long range lookups depends on the selectivity of the lookup range, and is $O(s \cdot N / B)$ for leveling and $O(s \cdot T \cdot N / B)$ for tiering. When answering range queries, tombstones and invalid entries have to be read and discarded, slowing down the range queries.

3.2.3 Write Amplification. Before being consolidated, an invalid entry may participate in multiple compactions. Repeatedly compacting invalid entries increases write amplification, which is particularly undesirable for installations that the durable storage has limited write endurance [58].

Analysis. We define write amplification, $w_{a m p}$ as the ratio of the total bytes written on disk that correspond to unmodified entries to the total bytes written corresponding to new or modified entries, $w_{a m p}=\frac{\operatorname{csize}\left(N^{+}\right)-\operatorname{csize}(N)}{\operatorname{csize}(N)} . N^{+}$is the number of all the entries written to disk including the entries re-written as unmodified after a compaction. For leveling, every entry participates on average in $T / 2$ compactions per level which makes $N^{+}=N \cdot L \cdot T / 2$. For tiering, every entry is written on disk once per level, implying $N^{+}=N \cdot L$. Thus, $w_{\text {amp }}$ for leveled and tiered LSM-trees are given by $O(L \cdot T)$ and $O(T)$, respectively. Note that, as the data size increases, entries participate in more compactions unmodified including invalid entries further increasing write amplification.

3.2.4 Persistence Latency and Data Privacy. The lack of guarantees in persistence latency has severe implications on data privacy. With new data privacy protection acts $[1,34]$

\begin{tabular}{|c|l|l|}
\hline Sym. & Description & Reference value \\
\hline$N$ & \# of entries inserted in tree (including tombstones) & $2^{20}$ entries \\
\hline$T$ & size ratio of tree & 10 \\
\hline$L$ & number of tree-levels on disk with $N$ entries & 3 levels \\
\hline$P$ & size of memory buffer in disk pages & 512 disk pages \\
\hline$B$ & number of entries in a disk page & 4 entries \\
\hline$E$ & average size of a key-value entry & 1024 bytes \\
\hline$M$ & memory buffer size & $16 \mathrm{MB}$ \\
\hline$\delta_{p}$ & number of point deletes issued & $3 \times 10^{5}$ entries \\
\hline$\delta_{r}$ & number of range deletes issued & $10^{3}$ entries \\
\hline$\sigma$ & average selectivity of range deletes & $5 \times 10^{-4}$ \\
\hline$N_{\delta}$ & approx. \# of entries after persisting deletes & - \\
\hline$\lambda$ & tombstone size / average key-value size & 0.1 \\
\hline$I$ & ingestion rate of unique entries in tree & 1024 entries/sec \\
\hline$S$ & selectivity of a long range lookup & - \\
\hline$L_{\delta}$ & number of tree-levels on disk with $N_{\delta}$ entries & - \\
\hline$m$ & total main memory allocated to BFs & $10 \mathrm{MB}$ \\
\hline$h$ & number of disk pages per delete tile & 16 disk pages \\
\hline
\end{tabular}

Table 1: Lethe parameters.

and the increased protection of rights like the right-to-beforgotten, data companies are legally obliged to persistently delete data offering guarantees [26] and rethink the end-toend data lifecycle [60].

Analysis. We define delete persistence latency as the worstcase time required, following the insertion of a tombstone, to ensure that the tree is void of any entry with a matching (older) key to that of the tombstone. This time depends on the insertion rate of unique key-value entries $(I)$ and the height of the tree $(L-1)$, and is the time needed to insert the minimum number of unique keys that is sufficient to trigger enough compactions. For leveling, delete persistence latency is $O\left(\frac{T^{L-1} \cdot P \cdot B}{I}\right)$ and for tiering is $O\left(\frac{T^{L} \cdot P \cdot B}{I}\right)$. This shows that for an LSM-tree with large number of entries $\left(T^{L}\right)$ that is built by an update-intensive workload, the delete persistence latency can be remarkably high.

\subsection{Implications of the Storage Layout}

Every file of an LSM-tree is sorted using the sort key. While this supports read, update, and delete queries on the sort key it cannot support operations on a secondary attribute.

Secondary Range Deletes on a delete key that is different from the sort key can only be supported by eagerly performing a full tree compaction, because there is no way to identify the affected files. This stalls all write operations, causing huge latency spikes. The cost incurred by a secondary range delete depends on the total number of data pages on disk, and is independent of the selectivity of the range delete operation. Irrespective of the merging strategy, this cost is $O(N / B)$, where $B$ is the page size.

\section{PERSISTING DELETES: LETHE}

Design Goals. Lethe aims (i) to provide persistence guarantees for point and range deletes and (ii) to enable practical secondary range deletes. We achieve the first design goal by introducing FADE, a family of delete-aware compaction strategies. We achieve the second goal by introducing Key 
Weaving Storage Layout, a new continuum of physical data layouts that arranges entries on disk in an interweaved fashion based on both the sort and the delete key.

\subsection{FADE}

We first introduce the $F A D E$ family of compaction strategies that ensures that all tombstones are persisted within a delete persistence threshold $\left(D_{t h}\right) . D_{t h}$ is typically specified by the application or user $[26,60]$ as part of the service level agreement (SLA) that concerns the data retention policy. All data streams bound by the same data retention SLA, have the same delete persistence latency.

4.1.1 Overview. Compactions in LSM-trees influence their behavior by dictating their space amplification, write amplification, point and range read performance, and delete persistence latency. FADE uses additional information about the age of a file's tombstones and the estimated invalidated entries per tombstone to ensure that every tombstone will adhere to the user/application-provided $D_{t h}$ by assigning to every file a time-to-live (TTL). As long as $D_{t h}$ is respected, FADE offers different strategies for secondary optimization goals including minimizing write amplification, minimizing space amplification, or maximizing system throughput.

4.1.2 Time-to-Live. To ensure that all delete operations issued on an LSM-tree are persisted before $D_{t h}$, FADE propagates each tombstone through all intermediate levels to the last level within that threshold from its insertion. FADE achieves this by assigning a smaller TTL for every file in every level $d_{i}$, such that $\sum_{i=0}^{L-1} d_{i}=D_{t h}$. A simple TTL allocation is to use $d_{i}=D_{t h} / L$. While this may guarantee that a tombstone reaches the last level within $D_{t h}$, it also leads to increased compaction time and resource starvation as larger levels have exponentially more files, hence, a large number of files may exhaust their TTL simultaneously. Instead, we assign exponentially increasing TTL per level to guarantee that files expire at a constant rate per time unit.

Computing $\boldsymbol{d}_{\boldsymbol{i}}$. For a tree with size ratio $T$, we set the TTL for level $i$ to be $d_{i}=T \cdot d_{i-1}, \forall i \in\{1, L-1\}$ and $d_{0}=$ $D_{t h} \cdot \frac{T-1}{T^{L-1}-1}$. Note that both the TTL and the capacity per level increase exponentially for larger levels of the tree.

Updating $\boldsymbol{d}_{\boldsymbol{i}}$. For a given tree height every file is assigned a TTL depending on the level it is being compacted into As more data entries are inserted, the tree might grow in height. At that point, the TTLs throughout the tree have to be updated. The cost of calculating $d_{i}$ is low, hence, FADE re-calculates $d_{i}$ after every buffer flush. Step 1 in Figure 4 (A) shows how to update $d_{i}$ when a new level is added.

4.1.3 FADE Metadata. Tombstones for point deletes are stored along with valid key-value pairs, and range tombstones are stored in a separate block. In addition to the tombstones, FADE requires the values of two metrics per file: (i) the age of the oldest tombstone contained $\left(a^{\max }\right)$ and (ii) the estimated invalidation count $(b)$ of the file tombstones. After every flush, a file is assigned with its current $a^{\max }$ and $b$.

In practice, LSM-engines store file metadata including (i) the file creation timestamp, and (ii) the distribution of the entries per file in the form of a histogram. For example, RocksDB assigns a monotonically increasing insertiondriven sequence number (seqnum) to all entries, and stores the number of entries (num_entries) and point tombstones (num_deletes) for every file. FADE takes advantage of this existing metadata. It uses seqnum to compute $a^{\max }$ and uses num_entries and num_deletes to compute $b$. Thus, in practice FADE leaves no metadata footprint.

Computing $\boldsymbol{a}^{\max }$. The $a^{\max }$ of a file $f$, termed $a_{f}^{\max }$, is the age of the oldest (point or range) tombstone contained in a file, and is calculated using the difference between the current system time and time the oldest tombstone of that file was inserted in the memory buffer. File without tombstones have $a_{f}^{\max }=0$. Storing $a_{f}^{\max }$ requires one timestamp (8 bytes) per file, a negligible overhead.

Computing $\boldsymbol{b}$. The estimated number of invalidated entries by the tombstones of a file $f$, termed $b_{f}$, is calculated using (i) the exact count of point tombstones in the file $\left(p_{f}\right)$ and (ii) an estimation of the entries of the entire database invalidated by the range tombstones of the file $\left(r d_{f}\right)$, as $b_{f}=p_{f}+r d_{f}$. It is not possible to accurately calculate $r d_{f}$ without accessing the entire database, hence, we estimate this value using the system-wide histograms that are already maintained by the data store. The value of $b_{f}$ is computed on-the-fly without needing any additional metadata.

Updating $\boldsymbol{a}^{\text {max }}$ and $\boldsymbol{b}$. Similarly to all file metadata, $a^{\max }$ and $b$ are first computed when a file is created after a buffer flush. Thereafter, for newly compacted files, $a^{\max }$ and $b$ are recomputed before they are written back to disk. When a compaction simply moves a file from one disk level to the next without physical sort-merging (i.e., when there are no overlapping keys), $b$ remains unchanged and $a^{\max }$ is recalculated based on the time of the latest compaction. Note that since all metadata is in memory, this does not cause an I/O.

4.1.4 Compaction Policies. Compactions ensure that both insert and read costs are amortized. For every compaction, there are two policies to be decided: the compaction trigger policy and the file selection policy. State-of-the-art LSM engines initiate a compaction when a level is saturated (i.e., larger than a nominal size threshold) and either pick a file at random, or the one with the smallest overlap with the subsequent level to minimize the merging cost.

Compaction Trigger. FADE augments the state-of-the-art by triggering a compaction, not only when a level is saturated, but also when a file has an expired TTL. FADE triggers a compaction in a level that has at least one file with expired 


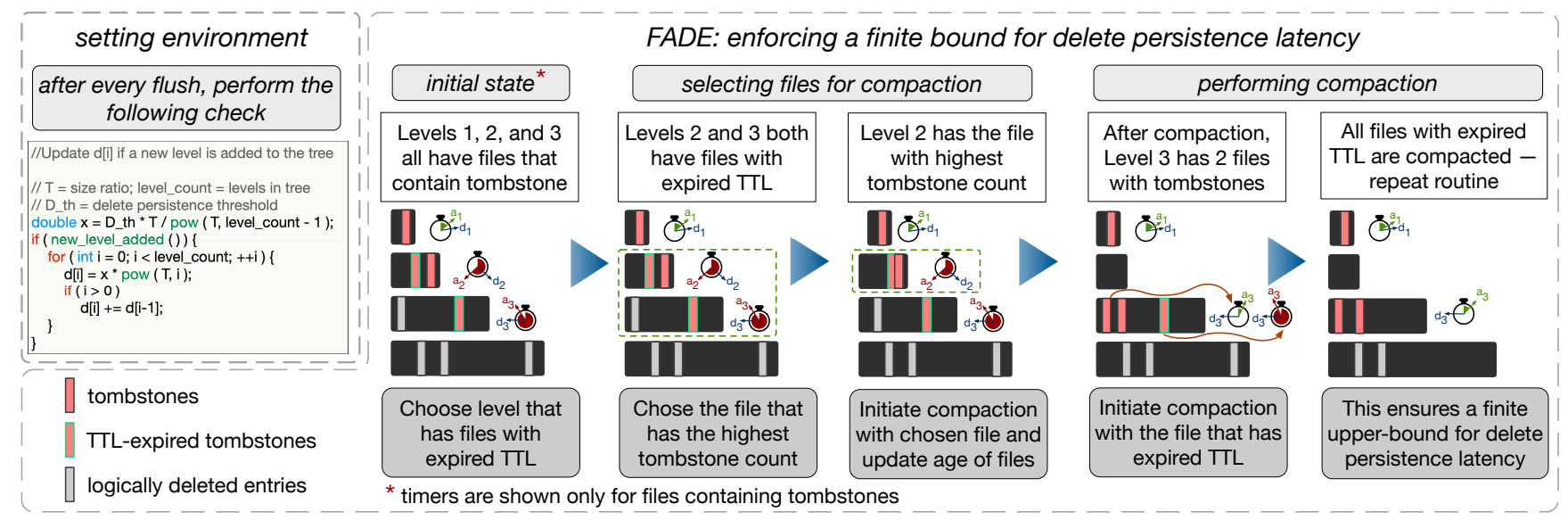

Figure 4: FADE persists tombstones within the delete persistence threshold, thus, improving overall performance.

TTL regardless of its saturation. If no TTL has expired, but a level is saturated, a compaction in that level is triggered.

File Selection. FADE decides which files to compact based on the trigger that invoked it. It has three modes: (i) the saturation-driven trigger and overlap-driven file selection (SO), which is similar to the state of the art and optimizes for write amplification, (ii) the saturation-driven trigger and deletedriven file selection (SD), which selects the file with the highest $b$ to ensure that as many tombstones as possible are compacted and to optimize for space amplification, and (iii) the delete-driven trigger and delete-driven file selection (DD), which selects a file with an expired tombstone to adhere to $D_{t h}$. A tie in SD and DD is broken by picking the file that contains the oldest tombstone, and a tie in SO by picking the file with the most tombstones. In case of a tie among levels, the smallest level is chosen for compaction to avoid write stalls during compaction. For a tie among files of the same level, FADE chooses the file with the most tombstones.

4.1.5 Implications on Performance. FADE guarantees that all point and range tombstones are persisted by the time their lifetime reaches $D_{t h}\left(\forall f, a_{f}^{\max }<D_{t h}\right)$. We refer to the size of the tree as $N$ and to the size of the tree that has all entries persisted within $D_{t h}$ as $N_{\delta}$.

Space amplification. FADE removes tombstones and logically invalidated entries from the tree on a rolling basis by compacting them in a time-bound fashion. By doing so, it diminishes the space amplification caused by out-of-place deletes, limiting $s_{a m p}$ to $O(1 / T)$ for leveling and $O(T)$ for tiering, even for a workload with deletes.

Write amplification. Ensuring delete persistence within $D_{t h}$, forces compactions on files with expired TTLs. Therefore, during a workload execution, initially FADE leads to momentary spikes in write amplification. The initial high write amplification, however, is amortized over time. By eagerly compacting tombstones, FADE purges most invalidated entries. Thus, future compactions involve fewer invalidated entries, leading to smaller write amplification which is comparable to the state of the art, as we show in Section 5.

Read performance. FADE has a marginally positive effect on read performance. By compacting invalidated entries and point tombstones, FADE reduces the number of entries hashed in the BFs, leading to smaller overall FPR for a given amount of available memory, hence, the cost for point lookups on existing and non-existing keys is improved asymptotically (Table 2 ). In the case that $N_{\delta}$ entries can be stored in $L_{\delta}<L$ levels on disk, the lookup cost will benefit by accessing fewer levels. Long range lookup cost is driven by the selectivity of the query, and this cost is lower for FADE as timely persistence of deletes causes the query iterator to scan fewer invalidated entries.

Persistence Guarantees. FADE ensures that all tombstones inserted into an LSM-tree and flushed to the disk will always be compacted with the last level within the user-defined $D_{t h}$ threshold. Any tombstone retained in the write-ahead $\log$ (WAL) is consistently purged if the WAL is purged at a periodicity that is shorter than $D_{t h}$, which is typically the case in practice. Otherwise, we use a dedicated routine that checks all live WALs that are older than $D_{t h}$, copies all live records to a new WAL, and discards the records in the older WAL that made it to the disk.

Practical Values for $D_{t h}$. The delete persistence threshold of different applications vary widely. In commercial systems, LSM-engines are forced to perform a full tree compaction every 7, 30, or 60 days based on the SLA requirements [39]. Blind Deletes. A tombstone against a key that does not exist or is already invalidated causes a blind delete. Blind deletes ingest tombstones against keys that do not exist in a tree, and these superfluous tombstones affect the performance of point and range queries [39]. To avoid blind deletes, FADE probes the corresponding $\mathrm{BF}$ and inserts a tombstone only if 


\begin{tabular}{|c|c|c|c|c|c|c|c|c|c|c|c|c|c|c|}
\hline \multirow{2}{*}{ Metric } & \multicolumn{2}{|c|}{ State-of-the-art $[21,23]$} & \multicolumn{3}{|c|}{ FADE } & & \multicolumn{4}{|c|}{ Key Weaving Storage Layout } & \multicolumn{4}{|c|}{ Lethe } \\
\hline & Leveling & Tiering & Leveling & & Tiering & & Leveling & & Tiering & & Leveling & & Tiering & \\
\hline Entries in tree & $O(N)$ & $O(N)$ & $O\left(N_{\delta}\right)$ & $\Delta$ & $O\left(N_{\delta}\right)$ & $\Delta$ & $O(N)$ & $\cdot$ & $O(N)$ & $\cdot$ & $O\left(N_{\delta}\right)$ & $\Delta$ & $O\left(N_{\delta}\right)$ & $\Delta$ \\
\hline Space amplification without deletes & $O(1 / T)$ & $O(T)$ & $O(1 / T)$ & $\cdot$ & $O(T)$ & $\cdot$ & $O(1 / T)$ & $\cdot$ & $O(T)$ & $\cdot$ & $O(1 / T)$ & $\cdot$ & $O(T)$ & $\cdot$ \\
\hline Space amplification with deletes & $O\left(\frac{(1-\lambda) \cdot N+1}{\lambda \cdot T}\right)$ & $O\left(\frac{N}{1-\lambda}\right)$ & $O(1 / T)$ & $\Delta$ & $O(T)$ & $\Delta$ & $O\left(\frac{(1-\lambda) \cdot N}{\lambda \cdot T}\right)$ & $\bullet$ & $O\left(\frac{N}{1-\lambda}\right)$ & $\cdot$ & $O(1 / T)$ & $\Delta$ & $O(T)$ & $\Delta$ \\
\hline Total bytes written to disk & $O(N \cdot E \cdot L \cdot T)$ & $O(N \cdot E \cdot L)$ & $O\left(N_{\delta} \cdot E \cdot L_{\delta} \cdot T\right)$ & $\Delta$ & $O\left(N_{\delta} \cdot E \cdot L_{\delta}\right)$ & $\Delta$ & $O(N \cdot E \cdot L \cdot T)$ & $\cdot$ & $O(N \cdot E \cdot L)$ & $\cdot 1$ & $O\left(N_{\delta} \cdot E \cdot L_{\delta} \cdot T\right)$ & $\Delta$ & $O\left(N_{\delta} \cdot E \cdot L_{\delta}\right)$ & $\Delta$ \\
\hline Write amplification & $O(L \cdot T)$ & $O(L)$ & $O(L \cdot T)$ & $\cdot$ & $O(L)$ & $\cdot$ & $O(L \cdot T)$ & $\cdot$ & $O(L)$ & $\cdot$ & $O(L \cdot T)$ & $\cdot$ & $O(L)$ & $\cdot$ \\
\hline Delete persistence latency & $O\left(\frac{T^{L-1} \cdot P \cdot B}{I}\right)$ & $O\left(\frac{T^{L} \cdot P \cdot B}{I}\right)$ & $O\left(D_{t h}\right)$ & $\Delta$ & $O\left(D_{t h}\right)$ & $\Delta$ & $O\left(\frac{T^{L-1} \cdot P \cdot B}{I}\right)$ & - & $O\left(\frac{T^{L} \cdot P \cdot B}{I}\right)$ & $\cdot$ & $O\left(D_{t h}\right)$ & $\Delta$ & $O\left(D_{t h}\right)$ & $\Delta$ \\
\hline Zero result point lookup cost & $O\left(e^{-m / N}\right)$ & $O\left(e^{-m / N} \cdot T\right)$ & $O\left(e^{-m / N_{\delta}}\right)$ & $\Delta$ & $O\left(e^{-m / N_{\delta}} \cdot T\right)$ & $\Delta$ & $O\left(h \cdot e^{-m / N}\right)$ & $\nabla$ & $O\left(h \cdot e^{-m / N} \cdot T\right)$ & $\mathbf{v}$ & $O\left(h \cdot e^{-m / N_{\delta}}\right)$ & 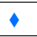 & $O\left(h \cdot e^{-m / N_{\delta}} \cdot T\right)$ & - \\
\hline Non-zero result point lookup cost & $O(1)$ & $O\left(1+e^{-m / N} \cdot T\right)$ & $O(1)$ & $\cdot$ & $O\left(1+e^{-m / N_{\delta}} \cdot T\right)$ & $\Delta$ & $O\left(1+h \cdot e^{-m / N}\right)$ & $\nabla$ & $O\left(1+h \cdot e^{-m / N} \cdot T\right.$ & T) $\mathbf{\nabla}$ & $O\left(1+h \cdot e^{-m / N_{\delta}}\right)$ & + & $O\left(1+h \cdot e^{-m / N_{\delta}} \cdot T\right)$ & + \\
\hline Short range point lookup cost & $O(L)$ & $O(L \cdot T)$ & $O\left(L_{\delta}\right)$ & $\Delta$ & $O\left(L_{\delta} \cdot T\right)$ & $\Delta$ & $O(h \cdot L)$ & $\nabla$ & $O(h \cdot L \cdot T)$ & $\nabla$ & $O\left(h \cdot L_{\delta}\right)$ & 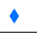 & $O\left(h \cdot L_{\delta} \cdot T\right)$ & $\checkmark$ \\
\hline Long range point lookup cost & $O\left(\frac{s \cdot N}{B}\right)$ & $O\left(\frac{T \cdot S \cdot N}{B}\right)$ & $O\left(\frac{s \cdot N_{\delta}}{B}\right)$ & $\Delta$ & $O\left(\frac{T \cdot s \cdot N_{\delta}}{B}\right)$ & $\Delta$ & $O\left(\frac{s \cdot N}{B}\right)$ & $\cdot$ & $O\left(\frac{T \cdot S \cdot N}{B}\right)$ & $\cdot$ & $O\left(\frac{s \cdot N_{\delta}}{B}\right)$ & $\Delta$ & $O\left(\frac{T \cdot s \cdot N_{\delta}}{B}\right)$ & $\Delta$ \\
\hline Insert/Update cost & $O\left(\frac{L \cdot T}{B}\right)$ & $O\left(\frac{L}{B}\right)$ & $O\left(\frac{L_{\delta} \cdot T}{B}\right)$ & $\Delta$ & $O\left(\frac{L_{\delta}}{B}\right)$ & $\Delta$ & $O\left(\frac{L \cdot T}{B}\right)$ & $\cdot$ & $O\left(\frac{L}{B}\right)$ & $\cdot$ & $O\left(\frac{L_{\delta} \cdot T}{B}\right)$ & $\Delta$ & $O\left(\frac{L_{\delta}}{B}\right)$ & $\Delta$ \\
\hline Secondary range delete cost & $O(N / B)$ & $O(N / B)$ & $O\left(N_{\delta} / B\right)$ & $\Delta$ & $O\left(N_{\delta} / B\right)$ & $\Delta$ & $O\left(\frac{N}{B \cdot h}\right)$ & $\Delta$ & $O\left(\frac{N}{B \cdot h}\right)$ & $\Delta$ & $O\left(\frac{N_{\delta}}{B \cdot h}\right)$ & $\bullet$ & $O\left(\frac{N_{\delta}}{B \cdot h}\right)$ & $\bullet$ \\
\hline Main memory footprint & $m+N \cdot \frac{k}{B}$ & $m+N \cdot \frac{k}{B}$ & $m+N_{\delta} \cdot\left(\frac{k}{B}+\frac{c}{B \cdot P}\right)$ & $\nabla$ & $m+N_{\delta} \cdot\left(\frac{k}{B}+\frac{c}{B \cdot P}\right)$ & $v$ & $m+N \cdot\left(\frac{1}{B \cdot h}\right)$ & $\Delta$ & $m+N \cdot\left(\frac{1}{B \cdot h}\right)$ & $\Delta$ & $m+N_{\delta} \cdot\left(\frac{k}{B \cdot h}+\frac{c}{B \cdot P}\right)$ & $\bullet$ & $m+N_{\delta} \cdot\left(\frac{k}{B \cdot h}+\frac{c}{B \cdot P}\right)$ & - \\
\hline
\end{tabular}

Table 2: Comparative analysis of state of the art and FADE ( $\Delta=$ better, $\nabla=$ worse, $\bullet=\operatorname{same}, \diamond=$ tunable).

the filter probe returns positive. This way, FADE drastically reduces the number of blind deletes.

\subsection{Key Weaving Storage Layout (KiWi)}

To facilitate secondary range deletes, we introduce $K i W i$, a continuum of physical storage layouts the arranges the data on disk in an interweaved sorted order on the sort key and delete key. KiWi supports secondary range deletes without performing a full-tree compaction, at the cost of minimal extra metadata and a tunable penalty on read performance. 4.2.1 The Layout. Figure 5 presents the internal structure of KiWi. Essentially, KiWi adds one new layer in the storage layout of LSM trees. In particular, in addition to the levels of the tree, the files of a level, and the page of a file, we now introduce delete tiles that belong to a file and consist of pages. In the following discussion, we use $\mathcal{S}$ to denote the sort key and $\mathcal{D}$ for the delete key.

Level Layout. The structure of the levels remains the same as in state-of-the-art LSM trees. Every level is a collection of files containing non-overlapping ranges of $\mathcal{S}$. The order between files in a level follows $\mathcal{S}$. Formally, if $i<j$, all entries in file $i$ have smaller $\mathcal{S}$ than those in file $j$.

File Layout. The contents of the file are delete tiles, which are collections of pages. Delete tiles contain non-overlapping ranges of $\mathcal{S}$, hence from the perspective of the file, the order of the delete tiles also follows $\mathcal{S}$. Formally, if $k<l$, all entries in delete tile $k$ have smaller $\mathcal{S}$ than those in file $l$.

Delete Tile Layout. Contrary to above, the pages of a delete tile are sorted on $\mathcal{D}$. Formally, for $p<q$, page $p$ of a given delete tile contains entries with smaller $\mathcal{D}$ than page $q$, while we have no information about $\mathcal{S}$. Organizing the contents of a tile ordered on the delete key $\mathcal{D}$ allows us to quickly execute range deletes because the entries under deletion are always clustered within contiguous pages of each tile, which can be dropped in their entirety.

Page layout. The order of entries within each page does not affect the performance of secondary range deletes, however, it significantly affects lookup cost, once a page is fetched to memory. To facilitate quick in-memory searches within a page [66], we sort the entries of each page based on $\mathcal{S}$.

4.2.2 Facilitating Secondary Range Deletes. KiWi exploits the fact that within a delete tile, the disk pages are sorted on the delete key. Hence, the entries targeted by a secondary range delete populate contiguous pages of each tile (in the general case of every tile of the tree). The benefit of this approach is that these pages can be dropped without having to be read and updated. Rather, they are removed from the otherwise immutable file and released to be reclaimed by the underlying file system. We call this a full page drop. Pages containing entries at the edge of the delete range, might also contain some valid entries. These pages are loaded and the valid entries are identified with a tight for-loop on $\mathcal{D}$ (since they are sorted on $\mathcal{S}$ ). The cost of reading and re-writing these pages is the $\mathrm{I} / \mathrm{O}$ cost of secondary range deletes with KiWi when compared with a full-tree compaction for the state of the art. We call these partial page drops, and constitute a small fraction of in-place editing, which is limited to zero or one page per delete tile. Subsequent compactions will create files and delete tiles with the pre-selected sizes.

4.2.3 Tuning and Metadata. We now discuss the tuning knobs and the metadata of KiWi.

Delete Tile Granularity. Every file contains a number of delete tiles, and each tile contains a number of pages. The basic tuning knob of KiWi is the number of pages per delete tile $(h)$, which affects the granularity of delete ranges that can be supported. For a file with $P$ disk pages, the number of delete tiles per file is $P / h$. The smallest granularity of a delete tile is when it consists of only a single disk page, i.e., for $h=1$. In fact, $h=1$ creates the same layout as the state of the art, as all contents are sorted on $\mathcal{S}$ and every range delete needs to update all data pages. As $h$ increases, delete ranges with delete fraction close to $1 / h$ will be executed mostly by full drops. On the other hand, for higher $h$ read 

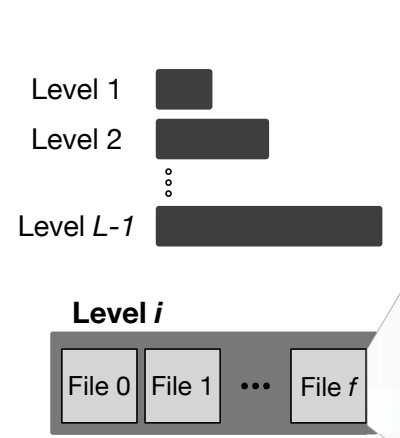

files in a level are sorted on sort key $(S)$ delete tiles within a file are sorted on $S$

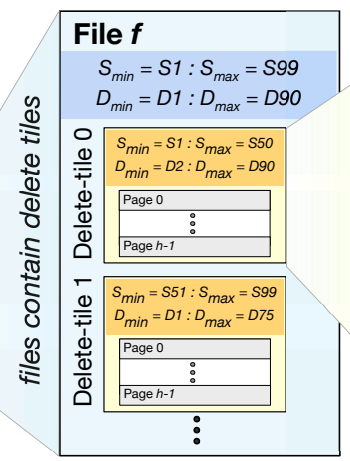

pages within a delete tile are sorted on delete key $(D)$

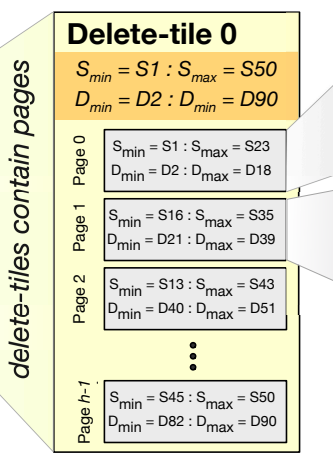

entries within every page are sorted on $S$

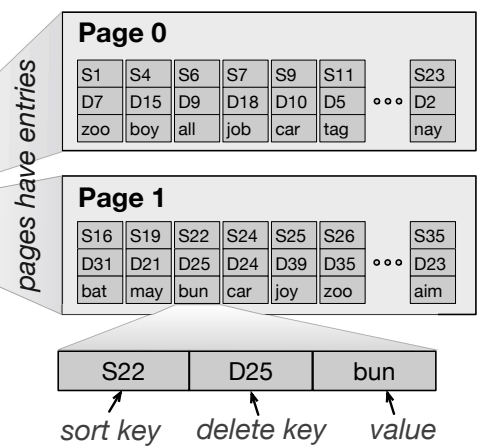

Figure 5: Key Weaving Storage Layout stores data in an interweaved fashion on the sort and delete key to facilitate efficient secondary range deletes without hurting read performance.

performance is affected. The optimal decision for $h$ depends on the workload (frequency of lookups and range deletes), and the tuning (memory allocated to BFs and size ratio).

Bloom Filters and Fence Pointers. We next discuss Bloom filters and fence pointers in the context of KiWi.

Bloom filters. KiWi maintains BFs on $\mathcal{S}$ at the granularity of disk page. Maintaining separate BFs per page requires no $\mathrm{BF}$ reconstruction for full page drops, and light-weight $\mathrm{CPU}$ cost for partial page drops. The same overall FPR is achieved with the same memory consumption when having one BF per page, since a delete tile contains no duplicates [10].

Fence pointers. KiWi maintains fence pointers on $\mathcal{S}$ that keep track of the smallest sort key for every delete tile. Fence pointers on $\mathcal{S}$, aided by the BFs, accelerate lookups. To support secondary range deletes, for every delete tile, KiWi maintains in memory a separate fence pointer structure on $\mathcal{D}$. We refer to this as delete fence pointers. The delete fence pointers store the smallest $\mathcal{D}$ of every page enabling full page drops of the corresponding pages without loading and searching the contents of a delete tile.

Memory Overhead. While KiWi does not require any additional memory for BFs, it maintains two fence pointer structures - one on $\mathcal{S}$ per delete tile and one on $\mathcal{D}$ per page. Since the state of the art maintains fence pointers on $\mathcal{S}$ per page, the space overhead for KiWi is the additional metadata per tile. Assuming sizeo $f(\mathcal{S})$ and sizeof $(\mathcal{D})$ are the sizes in bytes for $\mathcal{S}$ and $\mathcal{D}$ respectively, the space overhead is:

$$
\begin{gathered}
K i W i_{\text {mem }}-\operatorname{SoA} A_{\text {mem }}= \\
\frac{N}{B \cdot h} \cdot \operatorname{sizeof}(\mathcal{S})+\frac{N}{B} \cdot \operatorname{sizeof}(\mathcal{D})-\frac{N}{B} \cdot \operatorname{sizeof}(\mathcal{S})= \\
\text { \#delete_tiles } \cdot(\operatorname{sizeof}(\mathcal{S})+h \cdot(\operatorname{sizeof}(\mathcal{D})-\operatorname{sizeof}(\mathcal{S})))
\end{gathered}
$$

Note that if sizeof $(\mathcal{S})=\operatorname{sizeof}(\mathcal{D})$ the overhead is only one sort key per tile, and if sizeo $f(\mathcal{D})<\operatorname{sizeo} f(\mathcal{S})$, KiWi might lead to less overall size of metadata.

4.2.4 CPU Overhead. KiWi navigates an intrinsic tradeoff between the CPU cost for additional hashing for Bloom filters and the I/O cost associated with data movement to and from disk. For non-zero result point queries, KiWi performs $L \cdot h / 4$ times more hash calculations compared to the state of the art, and $L \cdot h$ times in case of zero-result point queries. In practice, commercial LSM engines, such as RocksDB, use only a single MurmurHash hash digest to calculate which Bloom filter bits to set $[58,69]$. This reduces the overall cost of hash calculation by almost one order of magnitude. We measured the time to hash a single 64-bit key using the MurmurHash to be $80 \mathrm{~ns}$, which is significantly smaller than the SSD access latency of $100 \mu$ s. This allows Lethe to strike a navigable trade-off between the CPU and I/O costs, and for the optimal value of $h$, Lethe achieves a significantly superior overall performance as compared to the state of the art.

4.2.5 Implications on Performance. KiWi offers a tunable trade-off between the cost for secondary range deletes and that of lookups, but does not influence write performance (including space and write amplifications).

Point Lookup. A point read follows the same search algorithm as in the state of the art [22]. In every level, a lookup searches the fence pointers (on $\mathcal{S}$ ) to locate the delete tile that may contain the search key. Once a delete tile is located, the $\mathrm{BF}$ for each delete tile page is probed. If a probe returns positive, the page is read to memory and binary searched, since the page is sorted on $\mathcal{S}$. If the key is found, the query terminates. If not, the $\mathrm{I} / \mathrm{O}$ was due to a false positive, and the next page of the tile is fetched. The $\mathrm{I} / \mathrm{O}$ cost for a query on an existing entry is $O\left(1+h \cdot e^{-m / N}\right)$ for leveling and $O\left(1+T \cdot h \cdot e^{-m / N}\right)$ for tiering. For a zero-result lookup the expected cost is $O\left(h \cdot e^{-m / N}\right)$ and $O\left(h \cdot e^{-m / N} \cdot T\right)$ respectively. Range Lookup. In general, a range lookup may span several delete tiles spread in one or more consecutive files. KiWi affects the performance of range lookups only at the terminal delete tiles that contain the bounds on the range - all delete tiles in between that are subsumed by the range always need 
to be read to memory. For the terminal delete tiles, the lookup needs to scan on average $h / 2$ more pages per tile instead of only the qualifying pages. Thus, the cost for short range lookups for KiWi becomes $O(h \cdot L)$ for leveling and $O(h \cdot L \cdot T)$ for tiering. For long range lookups, the increase in cost gets amortized over the number of qualifying delete tiles, and remains the same asymptotically, i.e., $O\left(\frac{s \cdot N}{B}\right)$ and $O\left(\frac{T \cdot s \cdot N}{B}\right)$ for leveling and tiering, respectively.

Secondary Range Lookups. With the interweaved layout, KiWi can also support efficient range lookups on the delete key. While state-of-the-art LSM engines need to maintain a secondary index on the delete key, they still pay a heavy cost for scanning across many scattered pages. KiWi utilizes the ordering of the data on the delete key and can realize secondary range queries at a much lower I/O cost.

4.2.6 Navigable Design. A fundamental design goal for KiWi is to navigate the trade-off between the cost of secondary range deletes and lookups. KiWi offers a navigable continuum of storage layouts that can be tuned to obtain the optimal value for $h$ based on the workload characteristics and performance requirements. Assuming that the workload can be described by the fractions of (a) point queries with zero-result $f_{E P Q}$, (b) point queries with non-zero result $f_{P Q}$, (c) short range queries $f_{S R Q}$, (d) long range queries $f_{L R Q}$ with selectivity $s$, (e) secondary range deletes $f_{S R D}$, and (f) insertions $f_{I}$, then we can compare the cost of this workload for Lethe and the state of the art.

$$
\begin{array}{r}
\text { Cost }_{\text {Lethe }} \leq \text { Cost }_{S o A} \Rightarrow f_{E P Q} \cdot F P R \cdot h+f_{P Q} \cdot(1+\cdot F P R \cdot h)+ \\
f_{S R Q} \cdot L_{\delta} \cdot h+f_{L R Q} \cdot s \cdot N / B+f_{S R D} \cdot N /(B \cdot h)+f_{I} \cdot \log _{T}(N / B) \leq \\
f_{E P Q} \cdot F P R+f_{P Q} \cdot(1+\cdot F P R)+f_{S R Q} \cdot L \cdot+f_{L R Q} \cdot s \cdot \frac{N}{B}+ \\
f_{S R D} \cdot N / B+f_{I} \cdot \log _{T}(N / B) \Rightarrow \\
\left(f_{E P Q}+f_{P Q}\right) \cdot F P R \cdot h+f_{S R Q} \cdot L_{\delta} \cdot h+f_{S R D} \cdot N /(B \cdot h) \leq \\
\left(f_{E P Q}+f_{P Q}\right) \cdot F P R+f_{S R Q} \cdot L+f_{S R D} \cdot N / B
\end{array}
$$

If we divide both sides of Eq. 1 with $f_{S R D}$ we will get the costs with respect to the relative frequencies of each operation with respect to the range deletes.

$$
\begin{array}{r}
(1) \Rightarrow\left(f_{E P Q}+f_{P Q}\right) / f_{S R D} \cdot F P R \cdot(h-1)+f_{S R Q} / f_{S R D} \cdot\left(L_{\delta} \cdot h-L\right) \leq \\
N / B \cdot(h-1) / h\left(\text { assuming } L_{\delta} \approx L\right) \\
\Rightarrow \frac{f_{E P Q}+f_{P Q}}{f_{S R D}} \cdot F P R+\frac{f_{S R Q}}{f_{S R D}} \cdot L \leq \frac{N}{B} \cdot \frac{1}{h}
\end{array}
$$

Using Eq. 2 we navigate the secondary range delete vs. lookup performance trade-off to find the best layout.

\subsection{Lethe}

Lethe puts together the benefits of FADE and KiWi to better support deletes in LSM-trees. For a given workload and a persistence delete latency threshold, Lethe offers maximal performance, by carefully tuning the cost of persistent deletes, and their impact on the overall performance of the system. The key tuning knobs are (i) the delete persistence threshold $\left(D_{t h}\right)$ and (ii) delete tile granularity $(h)$. The delete persistence threshold is specified as part of the data retention SLA, and Lethe sets the TTL for the tree-levels to ensure timely persistence.

For a workload with secondary range deletes, Lethe tunes the storage layout to find the optimal value for the delete tile granularity using the frequency of read operations relative to the frequency of secondary range deletes. The optimal value of $h$ is given by solving Eq. 2 with respect to $h$.

$$
h \leq \frac{N}{B} \cdot \frac{1}{\frac{f_{E P Q}+f_{P Q}}{f_{S R D}} \cdot F P R+\frac{f_{S R Q}}{f_{S R D}} \cdot L}
$$

For example, for a $400 \mathrm{~GB}$ database and $4 \mathrm{~KB}$ page size, if between two range deletes we execute $50 \mathrm{M}$ point queries of any type, $10 \mathrm{~K}$ short range queries, and have $F P R \approx 0.02$ and $T=10$, using Eq. 3, we have the optimal value of $h$ as:

$$
h \leq \frac{400 G B / 4 K B}{5 \cdot 10^{7} \cdot 2 \cdot 10^{-2}+10^{4} \cdot \log _{10}\left(\frac{400 G B}{4 K B}\right)}=\frac{10^{8}}{10^{6}+8 \cdot 10^{4}} \approx 10^{2} .
$$

Implementation. Lethe is implemented on top of RocksDB which is an open-source LSM-based key-value store widely used in the industry [27, 29]. The current implementation of RocksDB is implemented as leveling (only Level 1 is implemented as tiered to avoid write-stalls) and has a fixed size ratio (defaults to 10). We develop a new API for Lethe to have fine-grained control on the infrastructure. The API allows us to initiate compactions in RocksDB based on custom triggers and design custom file picking policies during compactions. RocksDB already stores metadata for every file, which includes the number of entries and deletes per files. We further store age as the only additional information per file. The delete persistence threshold is taken as a user-input at setup time and is used to dynamically set the level-TTLs.

\section{EVALUATION}

We evaluate Lethe against state-of-the-art LSM-tree designs for a range of workloads with deletes and different delete persistence thresholds.

Experimental Setup. We use a server with two Intel Xeon Gold $62302.1 \mathrm{GHz}$ processors each having 20 cores with virtualization enabled and with 27.5MB L3 cache, 384GB of RDIMM main memory at $2933 \mathrm{MHz}$ and 240GB SSD.

Default Setup. Unless otherwise mentioned the experimental setup consists of an initially empty database with ingestion rate at $2^{10}$ entries per second. The size of each entry is $1 \mathrm{~KB}$, and the entries are uniformly and randomly distributed across the key domain and are inserted in random order. The size of the memory buffer is $1 \mathrm{MB}$ (implemented as a skiplist). The size ratio for the database is set to 10 , and for the Bloom filters in memory, we use 10 bits per entry. To determine the raw performance, write operations are considered to have a lower priority than compactions. For all experiments 
performed, the implementation for Lethe differed from the RocksDB setup in terms of only the compaction trigger and file picking policy. We have both block cache and direct I/O enabled and the WAL disabled. Deletes are issued only on keys that have been inserted in the database and are uniformly distributed within the workload. We insert $1 \mathrm{~GB}$ data in the database with compactions given a higher priority than writes. The delete persistence threshold is set to $16.67 \%$, $25 \%$, and $50 \%$ of the experiment's run-time. This experiment mimics the behavior of a long-running workload. The delete persistence threshold values chosen for experimentation are representative of practical applications, where the threshold is 2 months (16.67\%), 3 months (25\%), 6 months (50\%), respectively, for a commercial database running for 1 year [26]. All lookups are issued after the whole database is populated.

Metrics. The compaction related performance metrics including (i) total number of compactions performed, (ii) total bytes compacted, (iii) number of tombstones present in a tree, and the (iv) age of files containing tombstones are measured by taking a snapshot of the database after the experiment. Space and write amplification are then computed using the equations from Sections 3.2.1 and 3.2.3.

Workload. Given the lack of delete benchmarks, we designed a synthetic workload generator, which produces a variation of YCSB Workload A, with $50 \%$ general updates and $50 \%$ point lookups. In our experiments, we vary the percentage of deletes between $2 \%$ to $10 \%$ of the ingestion.

\subsection{Achieving Timely Delete Persistence}

Lethe Reduces Space Amplification. We first show that Lethe significantly reduces space amplification by persisting deletes timely. We set up this experiment by varying the percentage of deletes in a workload for both RocksDB and Lethe, for three different delete persistence thresholds. The plot is shown in Figure 6 (A). For a workload with no deletes, the performances of Lethe and RocksDB are identical. This is because in the absence of deletes, Lethe performs compactions triggered by level-saturation, choosing files with minimal overlap. In the presence of deletes, driven by the delete persistence threshold $\left(D_{t h}\right)$, Lethe compacts files more frequently to ensure compliance with the threshold. It deletes the logically invalidated entries persistently, and in the process, diminishes the space amplification in the database. Even when $D_{t h}$ is set to $50 \%$ of the workload execution duration, Lethe reduces space amplification by about $48 \%$. For shorter $D_{t h}$, the improvements in space amplification are further pronounced by Lethe.

Lethe Performs Fewer Compactions. Figures 6 (B) and (C) show that Lethe performs fewer compactions as compared to RocksDB, but compacts more data during every compaction. Lethe performs compactions on a rolling basis based on $D_{t h}$.
After each experiment, Lethe was found to have fewer files on disk as compared to RocksDB. This is because, Lethe compacts invalidated entries in a greedy manner, and for a workload with even a small fraction (2\%) of deletes, it reduces the number of compactions performed by $45 \%$, as shown in Figure 6 (B). However, while compacting files with expired TTLs, the chosen file may overlap with a relatively higher number of files from the target level, and thus Lethe compacts $4.5 \%$ more data when $D_{t h}$ is set as $50 \%$ of the experiment's run-time, as shown in Figure 6 (C).

Lethe Achieves Better Read Throughput. In this experiment, we show that Lethe offers a superior read performance as compared to RocksDB. For this experiment, we populate the database with $1 \mathrm{~GB}$ data and then issue point lookups on existing entries. Note that the lookups may be on entries have been deleted by a tombstone after they were inserted. With more deletes in the workload, the number of invalidated entries (including tombstones) hashed into the BFs increases. Lethe purges these superfluous entries by persisting them in a time-bound manner, and thus, cleans up the BFs and improves their FPR. A lookup on a persistently deleted key returns negative without performing a disk $\mathrm{I} / \mathrm{O}$ to read a tombstone. Overall, Figure 6 (D) shows that Lethe improves lookup performance by up to $17 \%$ for workloads with deletes.

Lethe Ensures Timely Delete Persistence. Figure 6 (E) shows the distribution of the tombstones ages at the end of the experiment to demonstrate that Lethe ensures timely persistent deletion. The X-axis shows the age of all files that contain tombstones, and the Y-axis shows the cumulative number of tombstones at the instant of the snapshot with the age corresponding to the $\mathrm{X}$-axis value or smaller. The goal of Lethe is to have fewer tombstones of smaller age than the state of the art, with all tombstones having age less than $D_{t h}$ We show that in comparison with RocksDB, Lethe persists between $40 \%$ and $80 \%$ more tombstones, and does so while honoring the delete persistence threshold. For $D_{t h}=50 \%$ of the experiment's run-time, while RocksDB has $\sim 40,000$ tombstones (i.e., $\sim 40 \%$ of all tombstones inserted) distributed among files that are older than $D_{t h}$, Lethe persists all deletes within the threshold.

Write Amplification gets Amortized for Lethe. This experiment demonstrates that the higher write amplification caused by the initial eager merging of Lethe is amortized over time. For Lethe, we set $D_{t h}$ to 60 seconds and take snapshots at an interval of 180 seconds during the execution of the experiments. At every snapshot, we measure the cumulative bytes written over the past intervals. We measure the same metric for RockDB (that does not support setting a $D_{t h}$ ), and use it to normalize the bytes written. We plot the normalized bytes written against time (across snapshots) in Figure $6(\mathrm{~F})$. We observe that due to eager merging, Lethe writes 


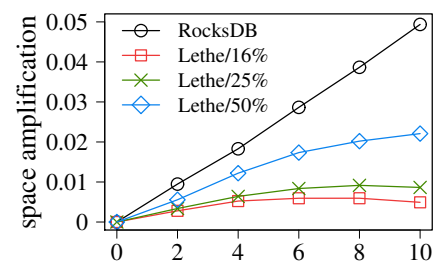

(A) $\%$ deletes in workload

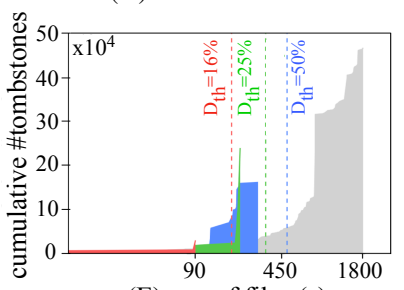

(E) age of files (s)

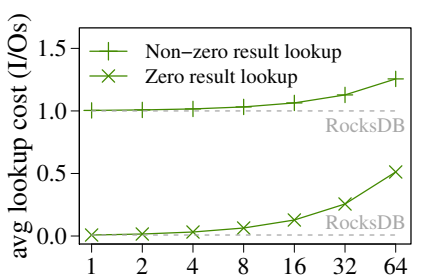

(I) delete-tile granularity (log scale)

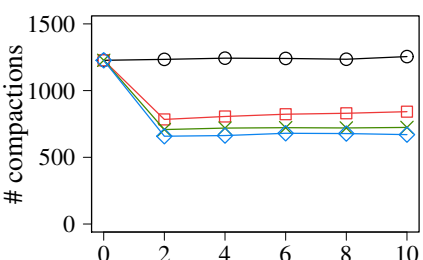

(B) $\%$ deletes in workload

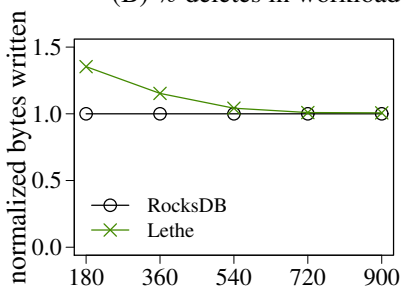

(F) time (s)

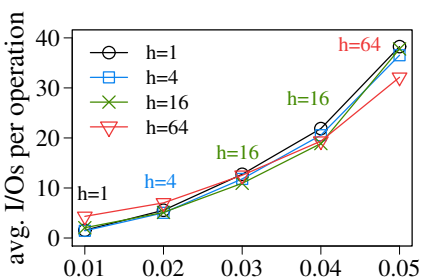

(J) fraction of deleted entries

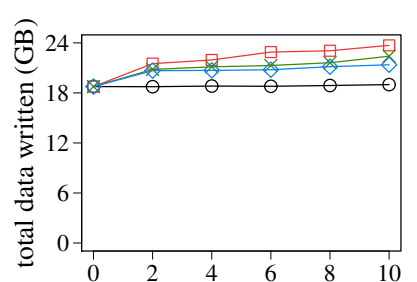

(C) \% deletes in workload

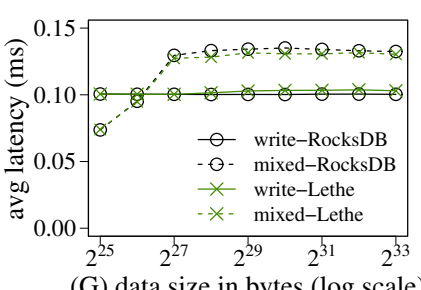

(G) data size in bytes (log scale)

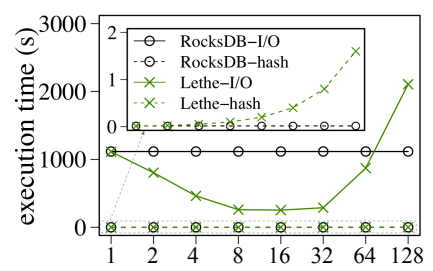

(K) delete tile size (pages)

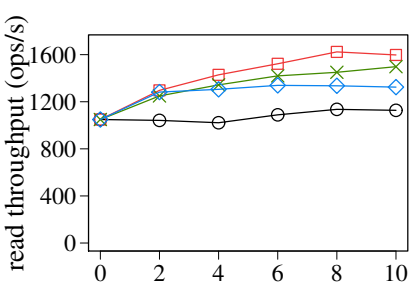

(D) $\%$ deletes in workload

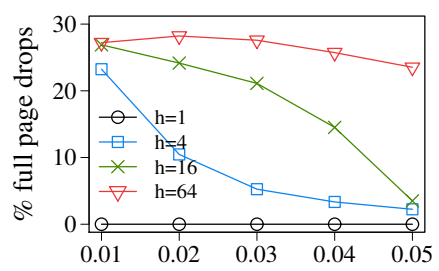

(H) fraction of deleted entries

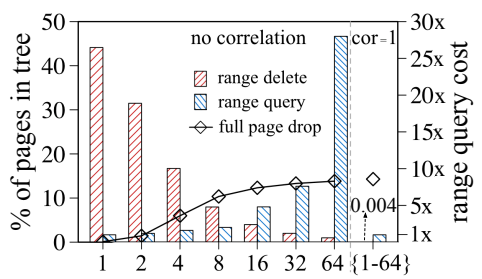

(L) delete tile size (pages)

Figure 6: Lethe improves space amplification (A) and performs fewer larger compactions (B, C) to persist deletes timely (E). In the process, it improves read throughput (D). The higher write amplification in Lethe gets amortized over time (F), which allows Lethe to scale similarly to RocksDB (G). (H) through (L) show Lethe's ability to navigate the design space of storage layouts and choose the optimal delete tile size to strike a balance between the lookup and secondary range delete performance to maximize the overall throughput.

$1.4 \times$ more data compared to RocksDB in the first snapshot. However, by persisting invalid entries upfront, Lethe purges superfluous entries from the tree, and hence, compacts fewer entries during subsequent compactions. This reduces the normalized writes by Lethe over time. At the end of the experiment, we observe that Lethe writes only $0.7 \%$ more data as compared to RocksDB. In this experiment, we set the $D_{t h}$ to be $15 \times$ smaller than the experiment duration to model the worst case. In practice, insertions in LSM engines continue for much longer (even perpetually) and $D_{t h}$ is set to a small constant duration. In this scenario Lethe's write amplification will be quickly amortized.

Lethe Scales Similarly to the State of the Art. This experiment shows that Lethe and the state of the art follow the same performance trends as data volume grows. We set up this experiment with the default configuration, and we vary data size. In addition to YCSB Workload A, which is used to compute the mixed workload latency, we use a write-only workload to measure write latency. Figure $6(G)$, shows the average latency for both workloads with data size on the $\mathrm{X}$-axis. We observe that the write latency for RocksDB and Lethe is not affected by data size. Due to the initial increased write amplification of Lethe, its write latency is $0.1-3 \%$ higher than that of RocksDB. For the mixed workload, however, Lethe improves the average latency by $0.5-4 \%$. This improvement is primarily due to the higher read throughput achieved by Lethe, as shown in Figure 6 (D). For smaller data sizes, most data entries are stored in memory or the first disk level, which reduces read latency significantly.

\subsection{Secondary Range Deletes}

Next, we evaluate secondary range deletes on Lethe.

Setup. Unless otherwise mentioned, the workload comprises $0.001 \%$ secondary range delete operations along with $1 \%$ range queries and $50 \%$ point queries. Each file has 256 pages and the size of every page is $4 \mathrm{~KB}$.

Lethe Achieves Superior Delete Performance. Figures $6(\mathrm{H})$ through $(\mathrm{L})$ show that Lethe offers superior overall performance by storing the data on disk using KiWi. For the first experiment, we vary the selectivity of a secondary range delete operation, i.e., the fraction of the database that is deleted, and measure the number of pages that can be fully dropped during the operation. Full page drops do not require reading the page to memory, and thus, a higher value along 
the Y-axis is desirable. We repeat the experiment for different delete tile granularity $(h)$. As the selectivity of the secondary range delete operation increases the number of full page drops decreases. This problem is further exacerbated for smaller delete tile granularity.

Although a higher value for $h$ is desirable for reducing the $\mathrm{I} / \mathrm{O}$ requirement for secondary range deletes, it bears a trade-off with the lookup performance, as shown in Figure 6 (I). The cost of zero-result and non-zero result lookups increases linearly with an increase in the tile size. Thus, the optimal value for $h$ is driven by the workload composition.

Choosing the Optimal Storage Layout. Figure 6 (J) shows Lethe's ability to navigate the continuum of storage layout and offer superior overall performance by determining the optimal storage layout. For a workload with a secondary range delete to lookup ratio of $2 \times 10^{-6}$ (i.e., 1 secondary range delete per $0.5 \mathrm{M}$ lookups), as the selectivity of the secondary range delete operation changes, the optimal way of storing data on disk changes. For selectivity $0.01 \%$, the classical storage layout (with $h=1$ ) provides optimal performance. As the selectivity increases, the design choice changes, and we observe that for selectivity $0.05 \%$ storing 64 disk pages per delete tile $(h=64)$ attains the optimal performance.

Analyzing the CPU-I/O Trade-off. In this experiment, we show the trade-off between the CPU and I/O costs for Lethe. The workload for this experiment has $50 \%$ point queries and $1 \%$ range query with selectivity of $0.001 \%, 49 \%$ inserts, and a single secondary range delete. We run this workload on a preloaded database of size $\sim 90 \mathrm{~GB}$ (i.e., $2^{10}$ inserts/sec for 24 hours). We have a single secondary range delete operation that deletes $1 / 7^{\text {th }}$ of the database (mimics the behavior of deleting all data older than 7 days). We measure the total time spent for hash computation for filter probes and the total time spent for I/Os to the disk.

Figure $6(\mathrm{~K})$ plots the total time spent in hashing and I/O access for both Lethe and RocksDB while varying delete tile size. The embedded figure shows that the hashing cost increases linearly with $h$. However, as the time to hash entries is 3 orders of magnitude smaller than the disk access latency and only point queries benefit from Bloom filters, the disk access time dominates the workload execution time. By design, Lethe computes the optimal value of $h$, which in this case is 8 . For $h=8$, the $\mathrm{I} / \mathrm{O}$ cost for Lethe is $76 \%$ lower than that in RocksDB. This comes at the price of a $5 \times$ increase in the hashing cost, which is completely hidden behind the massive benefits in total number of I/Os.

Effects of Correlation between Sort Key and Delete Key. Figure 6 (L) shows the effect of correlation between the sort key and the delete key. We run this experiment for two workloads. For the first workload, where there is no correlation between the sort and delete keys, the impact of the interweaved storage layout is prominent. As we increase $h$ the range delete cost drops drastically (because a larger fraction of pages can be fully dropped) at the expense of the cost of range queries. For the second workload, which has positive correlation between sort and delete key $(\approx 1)$, delete tiles have no impact on performance. For such a case, the classical LSM storage layout (i.e., $h=1$ ) becomes optimal.

\section{RELATED WORK}

Deletion in Relational Systems. Past work on data deletion on relational systems focuses on bulk deletes [14, 31, 47]. Efficient bulk deletion relies on similar techniques as efficient reads: sorting or hashing data to quickly locate, and ideally collocate, the entries to be deleted. Efficient deletion has also been studied in the context of spatial data [45] and view maintenance [8]. Contrary to past work, Lethe aims to support a user-provided delete persistence latency threshold. Self-Destructing Data. In addition, past research has proposed to automatically make data disappear when specific conditions are met. Vanish is a scheme that ensures that all copies of certain data become unreadable after a userspecified time, without any specific action on the part of a user [32, 33]. Kersten [42] and Heinis [37] have proposed the concept of forgetting in data systems through biologyinspired mechanisms as a way to better manage storage space and for efficient data analysis capabilities, as the data generation trends continue to increase. Contrary to this, Lethe supports timely data deletion that is set by users/applications.

\section{CONCLUSION}

In this work, we show that state-of-the-art LSM-based keyvalue stores perform suboptimally for workloads with even a small proportion of deletes, and that the delete persistence latency in these data stores are potentially unbounded. To address this, we build Lethe, a new LSM-based engine that introduces a new family of compaction strategies FADE and $\mathrm{KiWi}$, a continuum of physical data storage layouts. FADE enforces delete persistence within a user-defined threshold while increasing read throughput and reducing space amplification, at the expense of a modest increase in write amplification. KiWi offers efficient secondary range deletes, which can be tuned to outperform state of the art for a given workload.

Acknowledgments. We thank the reviewers for their valuable feedback and researchers from Facebook for their useful remarks. We are particularly thankful to Xuntao Cheng from Alibaba and Mark Callaghan from MongoDB for the valuable discussions and concrete feedback. We also thank Zichen Zhu for helping with the experimentation. This work was partially funded by NSF Grant No. IIS-1850202. 


\section{REFERENCES}

[1] California Consumer Privacy Act of 2018. Assembly Bill No. 375, Chapter 55, 2018.

[2] T. Akidau, R. Bradshaw, C. Chambers, S. Chernyak, R. FernándezMoctezuma, R. Lax, S. McVeety, D. Mills, F. Perry, E. Schmidt, and S. Whittle. The Dataflow Model: A Practical Approach to Balancing Correctness, Latency, and Cost in Massive-Scale, Unbounded, Out-of-Order Data Processing. Proceedings of the VLDB Endowment, 8(12):1792-1803, 2015.

[3] I. Alagiannis, S. Idreos, and A. Ailamaki. H2O: A Hands-free Adaptive Store. In Proceedings of the ACM SIGMOD International Conference on Management of Data, pages 1103-1114, 2014.

[4] Apache. Accumulo. https://accumulo.apache.org/.

[5] Apache. Cassandra. http://cassandra.apache.org.

[6] Apache. HBase. http://hbase.apache.org/.

[7] R. Appuswamy, M. Karpathiotakis, D. Porobic, and A. Ailamaki. The Case For Heterogeneous HTAP. In Proceedings of the Biennial Conference on Innovative Data Systems Research (CIDR), 2017.

[8] C. Aravindan and P. Baumgartner. A Rational and Efficient Algorithm for View Deletion in Databases. In Proceedings of the International Symposium on Logic Programming (ILPS), pages 165-179, 1997.

[9] J. Arulraj, A. Pavlo, and P. Menon. Bridging the Archipelago between Row-Stores and Column-Stores for Hybrid Workloads. In Proceedings of the ACM SIGMOD International Conference on Management of Data, pages 583-598, 2016.

[10] M. Athanassoulis and A. Ailamaki. BF-Tree: Approximate Tree Indexing. Proceedings of the VLDB Endowment, 7(14):1881-1892, 2014.

[11] M. Athanassoulis, S. Chen, A. Ailamaki, P. B. Gibbons, and R. Stoica. MaSM: Efficient Online Updates in Data Warehouses. In Proceedings of the ACM SIGMOD International Conference on Management of Data, pages 865-876, 2011.

[12] M. Athanassoulis, S. Chen, A. Ailamaki, P. B. Gibbons, and R. Stoica. Online Updates on Data Warehouses via Judicious Use of Solid-State Storage. ACM Transactions on Database Systems (TODS), 40(1), 2015.

[13] M. Athanassoulis, M. S. Kester, L. M. Maas, R. Stoica, S. Idreos, A. Ailamaki, and M. Callaghan. Designing Access Methods: The RUM Conjecture. In Proceedings of the International Conference on Extending Database Technology (EDBT), pages 461-466, 2016.

[14] B. Bhattacharjee, T. Malkemus, S. Lau, S. Mckeough, J.-A. Kirton, R. V. Boeschoten, and J. Kennedy. Efficient Bulk Deletes for Multi Dimensionally Clustered Tables in DB2. In Proceedings of the International Conference on Very Large Data Bases (VLDB), pages 1197-1206, 2007.

[15] M. Callaghan. Deletes are fast and slow in an LSM. http://smalldatum.blogspot.com/2020/01/deletes-are-fast-and-slow-inlsm.html, 2020.

[16] Z. Cao, S. Dong, S. Vemuri, and D. H. C. Du. Characterizing, Modeling, and Benchmarking RocksDB Key-Value Workloads at Facebook. In 18th USENIX Conference on File and Storage Technologies (FAST 20), pages 209-223, 2020.

[17] F. Chang, J. Dean, S. Ghemawat, W. C. Hsieh, D. A. Wallach, M. Burrows, T. Chandra, A. Fikes, and R. E. Gruber. Bigtable: A Distributed Storage System for Structured Data. In Proceedings of the USENIX Symposium on Operating Systems Design and Implementation (OSDI), pages 205-218, 2006.

[18] Cisco. Cisco Global Cloud Index: Forecast and Methodology, 2016-2021. White Paper, 2018.

[19] J. C. Corbett, J. Dean, M. Epstein, A. Fikes, C. Frost, J. J. Furman, S. Ghemawat, A. Gubarev, C. Heiser, P. Hochschild, W. C. Hsieh, S. Kanthak, E. Kogan, H. Li, A. Lloyd, S. Melnik, D. Mwaura, D. Nagle, S. Quinlan, R. Rao, L. Rolig, Y. Saito, M. Szymaniak, C. Taylor, R. Wang, and D. Woodford. Spanner: Google's Globally-Distributed Database. In
Proceedings of the USENIX Symposium on Operating Systems Design and Implementation (OSDI), pages 251-264, 2012.

[20] B. Dageville, T. Cruanes, M. Zukowski, V. Antonov, A. Avanes, J. Bock, J. Claybaugh, D. Engovatov, M. Hentschel, J. Huang, A. W. Lee, A. Motivala, A. Q. Munir, S. Pelley, P. Povinec, G. Rahn, S. Triantafyllis, and P. Unterbrunner. The Snowflake Elastic Data Warehouse. In Proceedings of the ACM SIGMOD International Conference on Management of Data, pages 215-226, 2016.

[21] N. Dayan, M. Athanassoulis, and S. Idreos. Monkey: Optimal Navigable Key-Value Store. In Proceedings of the ACM SIGMOD International Conference on Management of Data, pages 79-94, 2017.

[22] N. Dayan, M. Athanassoulis, and S. Idreos. Optimal Bloom Filters and Adaptive Merging for LSM-Trees. ACM Transactions on Database Systems (TODS), 43(4):16:1-16:48, 2018.

[23] N. Dayan and S. Idreos. Dostoevsky: Better Space-Time Trade-Offs for LSM-Tree Based Key-Value Stores via Adaptive Removal of Superfluous Merging. In Proceedings of the ACM SIGMOD International Conference on Management of Data, pages 505-520, 2018.

[24] N. Dayan and S. Idreos. The Log-Structured Merge-Bush \& the Wacky Continuum. In Proceedings of the ACM SIGMOD International Conference on Management of Data (SIGMOD), pages 449-466, 2019.

[25] G. DeCandia, D. Hastorun, M. Jampani, G. Kakulapati, A. Lakshman, A. Pilchin, S. Sivasubramanian, P. Vosshall, and W. Vogels. Dynamo: Amazon's Highly Available Key-value Store. ACM SIGOPS Operating Systems Review, 41(6):205-220, 2007.

[26] A. Deshpande and A. Machanavajjhala. ACM SIGMOD Blog: Privacy Challenges in the Post-GDPR World: A Data Management Perspective. http://wp.sigmod.org/?p=2554, 2018.

[27] S. Dong, M. Callaghan, L. Galanis, D. Borthakur, T. Savor, and M. Strum. Optimizing Space Amplification in RocksDB. In Proceedings of the Biennial Conference on Innovative Data Systems Research (CIDR), 2017.

[28] Facebook. MyRocks. http://myrocks.io/.

[29] Facebook. RocksDB. https://github.com/facebook/rocksdb.

[30] Gartner. Gartner Says 8.4 Billion Connected "Things" Will Be in Use in 2017, Up 31 Percent From 2016. https://tinyurl.com/Gartner2020, 2017.

[31] A. Gärtner, A. Kemper, D. Kossmann, and B. Zeller. Efficient Bulk Deletes in Relational Databases. In Proceedings of the IEEE International Conference on Data Engineering (ICDE), pages 183-192, 2001.

[32] R. Geambasu, T. Kohno, A. A. Levy, and H. M. Levy. Vanish: Increasing Data Privacy with Self-Destructing Data. In Proceedings of the USENIX Security Symposium, pages 299-316, 2009.

[33] R. Geambasu, A. A. Levy, T. Kohno, A. Krishnamurthy, and H. M. Levy. Comet: An active distributed key-value store. In Proceedings of the USENIX Symposium on Operating Systems Design and Implementation (OSDI), pages 323-336, 2010.

[34] M. Goddard. The EU General Data Protection Regulation (GDPR): European Regulation that has a Global Impact. International fournal of Market Research, 59(6):703-705, 2017.

[35] G. Golan-Gueta, E. Bortnikov, E. Hillel, and I. Keidar. Scaling Concurrent Log-Structured Data Stores. In Proceedings of the ACM European Conference on Computer Systems (EuroSys), pages 32:1-32:14, 2015.

[36] Google. LevelDB. https://github.com/google/leveldb/.

[37] T. Heinis and A. Ailamaki. Reconsolidating Data Structures. In Proceedings of the International Conference on Extending Database Technology (EDBT), pages 665-670, 2015.

[38] S. Héman, M. Zukowski, and N. J. Nes. Positional Update Handling in Column Stores. In Proceedings of the ACM SIGMOD International Conference on Management of Data, pages 543-554, 2010.

[39] G. Huang, X. Cheng, J. Wang, Y. Wang, D. He, T. Zhang, F. Li, S. Wang, W. Cao, and Q. Li. X-Engine: An Optimized Storage Engine for Largescale E-commerce Transaction Processing. In Proceedings of the ACM 
SIGMOD International Conference on Management of Data, pages 651665, 2019.

[40] Y. Huang, T. Z. J. Fu, D.-M. Chiu, J. C. S. Lui, and C. Huang. Challenges, design and analysis of a large-scale p2p-vod system. In Proceedings of the ACM SIGCOMM 2008 Conference on Applications, Technologies, Architectures, and Protocols for Computer Communications, Seattle, WA, USA, August 17-22, 2008, pages 375-388, 2008.

[41] F. Hueske. State TTL for Apache Flink: How to Limit the Lifetime of State. Ververica, 2018.

[42] M. L. Kersten. Big Data Space Fungus. In Proceedings of the Biennial Conference on Innovative Data Systems Research (CIDR), Gong show talk, 2015.

[43] S. Kulkarni, N. Bhagat, M. Fu, V. Kedigehalli, C. Kellogg, S. Mittal, J. M. Patel, K. Ramasamy, and S. Taneja. Twitter Heron: Stream Processing at Scale. In Proceedings of the ACM SIGMOD International Conference on Management of Data, pages 239-250, 2015.

[44] A. Lamb, M. Fuller, and R. Varadarajan. The Vertica Analytic Database: C-Store 7 Years Later. Proceedings of the VLDB Endowment, 5(12):17901801, 2012.

[45] J.-T. Lee and G. G. Belford. An Efficient Object-based Algorithm for Spatial Searching, Insertion and Deletion. In Proceedings of the IEEE International Conference on Data Engineering (ICDE), pages 40-47, 1992.

[46] Y. Li, B. He, J. Yang, Q. Luo, K. Yi, and R. J. Yang. Tree Indexing on Solid State Drives. Proceedings of the VLDB Endowment, 3(1-2):1195-1206, 2010.

[47] T. Lilja, R. Saikkonen, S. Sippu, and E. Soisalon-Soininen. Online Bulk Deletion. In Proceedings of the IEEE International Conference on Data Engineering (ICDE), pages 956-965, 2007.

[48] LinkedIn. Voldemort. http://www.project-voldemort.com.

[49] H. Lu. Peer-to-Peer Support for Massively Multiplayer Games. In Proceedings IEEE INFOCOM 2004, The 23rd Annual foint Conference of the IEEE Computer and Communications Societies, Hong Kong, China, March 7-11, 2004, 2004.

[50] C. Luo and M. J. Carey. LSM-based Storage Techniques: A Survey. The VLDB fournal, 2019.

[51] C. Mohan. Hybrid Transaction and Analytics Processing (HTAP): State of the Art. In Proceedings of the International Workshop on Business Intelligence for the Real-Time Enterprise (BIRTE), 2016.

[52] MongoDB. Online reference. http://www.mongodb.com/.

[53] P. E. O'Neil, E. Cheng, D. Gawlick, and E. J. O'Neil. The log-structured merge-tree (LSM-tree). Acta Informatica, 33(4):351-385, 1996.

[54] F. Özcan, Y. Tian, and P. Tözün. Hybrid Transactional/Analytical Processing: A Survey. In Proceedings of the ACM SIGMOD International Conference on Management of Data, pages 1771-1775, 2017.

[55] A. Pantelopoulos and N. G. Bourbakis. Prognosis: a wearable healthmonitoring system for people at risk: methodology and modeling. IEEE
Trans. Information Technology in Biomedicine, 14(3):613-621, 2010.

[56] S. Papadopoulos, K. Datta, S. Madden, and T. Mattson. The TileDB Array Data Storage Manager. Proceedings of the VLDB Endowment, 10(4):349-360, 2016.

[57] P. Raju, R. Kadekodi, V. Chidambaram, and I. Abraham. PebblesDB: Building Key-Value Stores using Fragmented Log-Structured Merge Trees. In Proceedings of the ACM Symposium on Operating Systems Principles (SOSP), pages 497-514, 2017.

[58] RocksDB. DeleteRange: A New Native RocksDB Operation. https://rocksdb.org/blog/2018/11/21/delete-range.html, 2018.

[59] T. Sakaki, M. Okazaki, and Y. Matsuo. Earthquake shakes Twitter users: real-time event detection by social sensors. In Proceedings of the 19th International Conference on World Wide Web, WWW 2010, Raleigh, North Carolina, USA, April 26-30, 2010, pages 851-860, 2010.

[60] S. Sarkar, J.-P. Banâtre, L. Rilling, and C. Morin. Towards Enforcement of the EU GDPR: Enabling Data Erasure. In Proceedings of the IEEE International Conference of Internet of Things (iThings), pages 1-8, 2018.

[61] R. Sears and R. Ramakrishnan. bLSM: A General Purpose Log Structured Merge Tree. In Proceedings of the ACM SIGMOD International Conference on Management of Data, pages 217-228, 2012.

[62] SQLite4. Online reference. https://sqlite.org/src4/.

[63] M. Stonebraker, D. J. Abadi, A. Batkin, X. Chen, M. Cherniack, M. Ferreira, E. Lau, A. Lin, S. R. Madden, E. J. O’Neil, P. E. O’Neil, A. Rasin, N. Tran, and S. Zdonik. C-Store: A Column-oriented DBMS. In Proceedings of the International Conference on Very Large Data Bases (VLDB), pages 553-564, 2005.

[64] R. Thonangi and J. Yang. On Log-Structured Merge for Solid-State Drives. In Proceedings of the IEEE International Conference on Data Engineering (ICDE), pages 683-694, 2017.

[65] Q.-C. To, J. Soto, and V. Markl. A Survey of State Management in Big Data Processing Systems. CoRR, abs/1702.0, 2017.

[66] P. Van Sandt, Y. Chronis, and J. M. Patel. Efficiently Searching InMemory Sorted Arrays: Revenge of the Interpolation Search? In Proceedings of the ACM SIGMOD International Conference on Management of Data, pages 36-53, 2019.

[67] Z. Whittaker and N. Lomas. Even years later, Twitter doesn't delete your direct messages. https://techcrunch.com/2019/02/15/twitter-directmessages/, 2019.

[68] WiredTiger. Source Code. https://github.com/wiredtiger/wiredtiger.

[69] H. Zhang, H. Lim, V. Leis, D. G. Andersen, M. Kaminsky, K. Keeton, and A. Pavlo. SuRF: Practical Range Query Filtering with Fast Succinct Tries. In Proceedings of the ACM SIGMOD International Conference on Management of Data, pages 323-336, 2018.

[70] M. Zukowski and P. A. Boncz. Vectorwise: Beyond Column Stores. IEEE Data Engineering Bulletin, 35(1):21-27, 2012. 